The University of Maine

DigitalCommons@UMaine

$9-20-2013$

\title{
Method for estimating mean particle size from high-frequency fluctuations in beam attenuation or scattering measurements
}

\author{
Nathan T. Briggs \\ University of Maine \\ Wayne H. Slade \\ Sequoia Scientific, Inc. \\ Emmanuel Boss \\ University of Maine, emmanuel.boss@maine.edu \\ Mary Jane Perry \\ University of Maine
}

Follow this and additional works at: https://digitalcommons.library.umaine.edu/sms_facpub

Part of the Oceanography and Atmospheric Sciences and Meteorology Commons

\section{Repository Citation}

Briggs, Nathan T.; Slade, Wayne H.; Boss, Emmanuel; and Perry, Mary Jane, "Method for estimating mean particle size from high-frequency fluctuations in beam attenuation or scattering measurements" (2013). Marine Sciences Faculty Scholarship. 164.

https://digitalcommons.library.umaine.edu/sms_facpub/164

This Article is brought to you for free and open access by DigitalCommons@UMaine. It has been accepted for inclusion in Marine Sciences Faculty Scholarship by an authorized administrator of DigitalCommons@UMaine. For more information, please contact um.library.technical.services@maine.edu. 


\title{
Method for estimating mean particle size from high-frequency fluctuations in beam attenuation or scattering measurements
}

\author{
Nathan T. Briggs,,${ }^{1, *}$ Wayne H. Slade, ${ }^{2}$ Emmanuel Boss, ${ }^{1}$ and Mary Jane Perry ${ }^{1}$ \\ ${ }^{1} S$ chool of Marine Sciences, University of Maine, Orono, Maine 04469, USA \\ ${ }^{2}$ Sequoia Scientific, Inc., 2700 Richards Rd., Bellevue, Washington 98005, USA \\ *Corresponding author: natebriggs@gmail.com
}

Received 9 May 2013; revised 4 August 2013; accepted 13 August 2013;

posted 14 August 2013 (Doc. ID 190152); published 13 September 2013

\begin{abstract}
The ability to estimate mean particle size using simple, low-power optical instruments promises to greatly expand coverage of particle size measurements in the ocean and advance understanding of myriad processes from sediment transport to biological carbon sequestration. Here we present a method for estimating the mean diameter of particles in suspension from high-resolution time series of simple optical measurements, such as beam attenuation or optical backscattering. Validation results from a laboratory clay aggregation experiment show a good fit with independent mean particle diameter estimates in the 10-80 $\mu \mathrm{m}$ diameter range, with relative biases of $17 \%-38 \%$ and relative root mean square errors of $10 \%-24 \%$. In the $80-200 \mu \mathrm{m}$ range, quantitative validation data were not available, but our mean diameter estimates correlated strongly with particle settling rates. (C) 2013 Optical Society of America

OCIS codes: （010.4450) Oceanic optics; (290.1350) Backscattering; (290.2200) Extinction; (290.5820)

Scattering measurements; (290.5850) Scattering, particles; (010.4458) Oceanic scattering.

http://dx.doi.org/10.1364/AO.52.006710
\end{abstract}

\section{Introduction}

Size is a fundamental characteristic of particles in the ocean, playing a critical role in how particles interact with each other and their environment. For example, organism size is a major determinant of ecosystem structure through its effects on metabolic rates and trophic interactions [1,2]. Particle size is also fundamental to biogeochemical cycling through its effect on sinking rate; large organic particles such as phytoplankton aggregates and fecal pellets sink rapidly, transporting carbon to the deep ocean and facilitating atmospheric $\mathrm{CO}_{2}$ drawdown [3-7].

Particle size is also critical to the understanding of sediment transport and deposition, primarily as a driver of sinking and aggregation rates within the

$1559-128 \mathrm{X} / 13 / 276710-16 \$ 15.00 / 0$

(C) 2013 Optical Society of America benthic boundary layer. These rates significantly affect dispersal patterns of sediments and associated nutrients, carbon, and contaminants in coastal systems. Improvement in understanding of these sediment processes has been a major aim of scientists and engineers hoping to improve prediction of the effects of dredging, contaminant release, and seasonal riverine and other physical processes on water quality in coastal regions [8-11].

Widespread measurements of particle size could therefore greatly advance our understanding of multiple oceanic processes, from phytoplankton ecology to $\mathrm{CO}_{2}$ drawdown to sediment contaminant transport. Many instruments, both bench-top and in situ, are used for measuring size of marine particles, including microscopes, electrozone (Coulter) counters, flow cytometers, cameras, and laser diffraction systems. However, the size, cost, ease of use, power, and data requirements of these technologies 
limit the systematic measurement of particle size in the ocean.

Shifrin $[12,13]$ developed an inversion method for estimating mean particle size in a water sample using fluctuations in beam transmissometer measurements caused by random distribution of particles. The low cost, moderate size, and widespread deployment of beam transmissometers make this an appealing method for expanding the coverage of particle size measurements in the ocean. However, Shifrin's inversion has received little attention in the oceanographic literature and has not, to our knowledge, been tested using commercially available equipment. Here we revisit Shifrin's inversion and present a similar but simpler inversion based on the variance-to-mean ratio (VMR; see Table 1 for a list of all abbreviations used in this paper) for estimating mean particle size using not only beam transmissometers, but also, unlike the Shifrin inversion, using smaller, low-power in situ backscattering and fluorescence sensors. Such sensors are already widely deployed on platforms such as moorings and autonomous floats and gliders, so the VMR inversion has the potential to greatly increase the coverage of in situ estimates of particle size at little or no added cost.

In Section $\underline{2}$ we present the VMR inversion, and in Section 3 we present validation data from a laboratory experiment, in which the particulate beam attenuation $c_{p}$ and particulate backscattering $b_{b p}$ (via scattering at $124^{\circ}$ ) of aggregating and settling clay particles were measured over time [14]. We use both the VMR and Shifrin inversions to convert the variability in $c_{p}$ to mean particle size and apply the VMR inversion to $b_{b p}$ as well. The estimated mean particle sizes vary by more than an order of

Table 1. Table of Terms

\begin{tabular}{|c|c|c|}
\hline Term & Units & Description \\
\hline $\bar{A}$ & $\mu \mathrm{m}^{2}$ & Area-weighted mean $A_{i}$ \\
\hline $\bar{A}_{\text {Shifrin }}$ & $\mu \mathrm{m}^{2}$ & $\bar{A}$ from the Shifrin inversion \\
\hline $\bar{A}_{\text {LISST }}$ & $\mu \mathrm{m}^{2}$ & $\bar{A}$ from the LISST PSD \\
\hline $\bar{A}^{*}$ & $\mu \mathrm{m}^{2}$ & Mean $A_{i}$ weighted by optical cross section \\
\hline$C$ & - & Particle beam attenuance \\
\hline$c$ & $\mathrm{~m}^{-1}$ & Beam attenuation coefficient \\
\hline$c_{p}$ & $\mathrm{~m}^{-1}$ & Particulate beam attenuation coefficient \\
\hline$\stackrel{p}{D_{i}}$ & $\mu \mathrm{m}$ & Equivalent circular diameter of $A_{i}$ \\
\hline $\bar{D}$ & $\mu \mathrm{m}$ & Equivalent circular diameter of $\bar{A}$ \\
\hline $\bar{D}_{c p}, \bar{D}_{b b p}$ & $\mu \mathrm{m}$ & $\bar{D}$ from the VMR inversion of $c_{p}$ and $b_{b p}$ \\
\hline$i$ & & Class of identical particles \\
\hline$N_{i}$ & - & Number of particles in class $i$ within the sample volume of an instrument \\
\hline$n_{\mathrm{VMR}}$ & - & Number of independent samples used to calculate the VMR \\
\hline PSD & & Particle size distribution \\
\hline$Q, Q_{b b}, Q_{c}$ & - & Optical efficiency: Fraction of incident light backscattered $\left(Q_{b b}\right)$ or attenuated $\left(Q_{c}\right)$ by a particle \\
\hline$R_{c p}$ & $\min ^{-1}$ & Relative rate of decrease in $c_{p}$ \\
\hline$S$ & $\mu \mathrm{m}^{2}$ & Cross sectional of a transmissometer beam (perpendicular to the beam) \\
\hline$s$ & - & Spectral slope of $\bar{D}_{c p}(\lambda)$ \\
\hline$t$ & $\mathrm{~s}$ & Time \\
\hline$t_{\mathrm{res}}$ & $\mathrm{s}$ & Particle residence time in $V$ \\
\hline$t_{\mathrm{samp}}$ & $\mathrm{s}$ & Integration time of a single measurement \\
\hline$T$ & - & Particle beam transmittance \\
\hline$\Delta t$ & $\mathrm{~s}$ & Lag time used for calculating autocorrelation \\
\hline$\lambda$ & $\mathrm{nm}$ & Wavelength of light (in air) \\
\hline$\tau$ & - & $t_{\mathrm{res}} / t_{\mathrm{samp}}$ \\
\hline
\end{tabular}


magnitude during the experiment and agree closely with both independent estimates of size and measures of particle settling throughout most of this size range, with the VMR inversion performing slightly more consistently than the Shifrin inversion. In Section 4, we discuss the accuracy of the VMR inversion and its potential for field application.

\section{Methods}

\section{A. VMR Inversion Equation}

The VMR inversion is defined in this section for a time series of the particulate beam attenuation coefficient $c_{p}$ but can equally be applied to other additive optical properties, such as $b_{b p}$. The VMR inversion is used to estimate a weighted mean cross-sectional area $\bar{A}$ of particles in suspension. $\bar{A}$ is formally defined in Eq. (1), where $t$ is time, $E\left[N_{i}(t)\right]$ is the mean number of particles of class $i$ within a sensor's sample volume and $A_{i}$ is the cross-sectional area of a particle of class $i$. The VMR inversion is given in Eq. (2), where $\bar{A}_{c p}$ is the VMR inversion estimate of $\bar{A}$, $\operatorname{Var}\left[c_{p}(t)\right]$ and $E\left[c_{p}(t)\right]$ are the variance and mean of $c_{p}(t), V$ is the sensor sample volume, $Q_{c}$ is the attenuation efficiency of a particle (also known as the extinction efficiency $Q_{\text {ext }}$ ), $\alpha(\tau)$ is a correction for water movement during the integration time of a single measurement $\left(t_{\text {samp }}\right)$, defined in Eq. (3), and $\tau$ is the ratio of particle residence time $t_{\text {res }}$ within the sample volume to $t_{\mathrm{samp}}$ [Eq. (4)]. The input parameters of Eq. (2) are described in detail in Subsections 2.A.1-2.Ā.4. Equations (1)-(4) are derived in Appendices $\mathrm{A}-\mathrm{C}$, given the assumptions that ambient particle concentration is constant over the period of a single VMR inversion calculation, particles are randomly distributed in space, all particles share the same $Q_{c}$ (see Subsection 4.E.1 and Appendix A), and random fluctuations of the number and size of particles in $V$ are the only source of variance in $c_{p}$. For ease of interpretation, we convert estimates of $\bar{A}$ into an equivalent diameter $\bar{D}$ by assuming a circular cross section [Eq. (ㅁ)]:

$$
\begin{gathered}
\bar{A}=\frac{\sum_{i} E\left[N_{i}(t)\right] A_{i}^{2}}{\sum_{i} E\left[N_{i}(t)\right] A_{i}}, \\
\bar{A}_{c p}=\frac{\operatorname{Var}\left[c_{p}(t)\right]}{E\left[c_{p}(t)\right]} \frac{V}{Q_{c}} \frac{1}{\alpha(\tau)}, \\
\alpha(\tau)=\left\{\begin{array}{ll}
1-(3 \tau)^{-1} & \text { if } \tau \geq 1 \\
\tau-\frac{\tau^{2}}{3} & \text { if } \tau \leq 1
\end{array},\right. \\
\tau=\left(\frac{t_{\text {res }}}{t_{\mathrm{samp}}}\right), \\
\bar{D}=2 \sqrt{\bar{A} \pi^{-1}} .
\end{gathered}
$$

1. Time Series Statistics, $\operatorname{Var}\left[c_{p}(t)\right]$ and $E\left[c_{p}(t)\right]$

The variance and mean are calculated over a finite period of time, during which changes in particle size and concentration should be minimal. If present, variance from sources other than the random distribution of particles in space should be removed to avoid a positive bias in the VMR inversion. Variance from low-frequency sources may be removed using a high-pass filter, as discussed in Subsection 2.C.2.

\section{Sample Volume, $V$}

If the response of the sensor to a particle within its sample volume is the same regardless of particle location (homogeneous), then $V$ is simply the sensor's geometric sample volume. We define $V$ for the nonhomogeneous case in Appendix B [Eq. (B7)].

\section{Attenuation Efficiency, $Q_{c}$}

The attenuation efficiency $Q_{c}$ is defined as the fraction of light incident on the cross-sectional area of a particle that is scattered away from the detector or absorbed. For the evaluation dataset presented here, we use two different values as estimates of $Q_{c}$ : first, literature values of $Q_{c}$ to simulate field applications of the VMR inversion in which independent size measurements would not be available; and second, we calculate $Q_{c}$ empirically, via Eq. (6) in order to isolate other potential sources of bias. Note that the VMR inversion assumes that all particles share the same $Q_{c}$, so that the mean $Q_{c}$ in Eq. (6) applies to all particles. Implications of variable $\bar{Q}_{c}$ are discussed in Subsection 4.E.1:

$$
Q_{c}=\frac{E\left[c_{p}(t)\right]}{\sum_{i} E\left[N_{i}(t)\right] A_{i} V^{-1}} .
$$

\section{Correction for Water Movement, $\alpha(\tau)$}

Movement of the sample relative to the sample volume during $t_{\mathrm{samp}}$ will increase the volume sampled, requiring a further correction factor $\alpha(\tau)$, given in

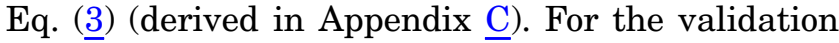
dataset presented here, movement of the sample was slow relative to $t_{\mathrm{samp}}$ for the ac-9 and ECO BB, so $\alpha(\tau)$ was close to one (Table 2). However, the longer averaging time settings of the LISST, especially during Exp. 2 cause $\alpha(\tau)$ to drop as low as 0.35 , becoming an important term in the VMR inversion.

\section{B. Evaluation Dataset}

The VMR inversion was tested using previously collected data from two laboratory clay aggregation experiments (Exp. 1 and Exp. 2) [14]. Setup and results of the two experiments were similar; however, during Exp. 1, a computer malfunction created a $10 \mathrm{~min}$ data gap, so the data presented in this section come from Exp. 2, unless noted otherwise. Initially, $4.2 \mathrm{~g}$ of bentonite clay were added to $120 \mathrm{~L}$ of reverse osmosis water in a $46-\mathrm{cm}$ deep, $100 \times 45 \mathrm{~cm}$ wide black 
Table 2. VMR Inversion Constant Input Parameters

\begin{tabular}{lcccccccc}
\hline Sensor $(\lambda, \mathrm{nm})$ & Experiment & $t_{\text {samp }}(\mathrm{s})$ & $t_{\text {res }}(\mathrm{s})$ & $\tau$ & $\alpha$ & $Q$ (Empirical) & $Q($ Literature) & $V(\mathrm{ml})$ \\
\hline ECO BBRT (660) & Exp. 1 & 0.87 & 5.6 & 6.4 & 0.95 & 0.01 & 0.024 \\
ECO BB3 (660) & Exp. 2 & 0.95 & 4.5 & 4.7 & 0.93 & 0.013 & 0.024 \\
ac-9 (650) & Exp. 1 & 0.003 & 4.3 & 1400 & 0.9998 & 1.53 & 0.62 \\
ac-9 (650) & Exp. 2 & 0.003 & 4.6 & 1500 & 0.9998 & 1.56 & 2 & 2 \\
LISST (670) & Exp. 1 & 4 & 3.8 & 0.95 & 0.65 & 1.68 & 5 \\
LISST (670) & Exp. 2 & 10 & 4 & 0.4 & 0.35 & 1.65 & 2 & 2 \\
\hline
\end{tabular}

laboratory sink. Subsequently, $47.6 \mathrm{~g}$ of $\mathrm{CaCl}_{2}$ salt dissolved in a small volume of water was added to the sink to induce aggregation; the water in the sink was stirred for $\sim 2$ min and not stirred thereafter. However, acoustic Doppler velocimeter measurements recorded $2-3 \mathrm{~mm} \mathrm{~s}^{-1}$ particle speeds for the remainder of the experiment (data not shown), most likely due to convection. During the course of the $4 \mathrm{~h}$ experiments, the particle size distribution (PSD) was estimated using a Sequoia Scientific LISST-100 (type $\mathrm{B}$; standard inversion; size range 1.4-230 $\mu \mathrm{m}$ diameter). Equation (1) was applied to the LISST PSD to calculate $\bar{A}_{\text {LISST }}$, which was then converted to an equivalent diameter $\bar{D}_{\text {LISST }}$ using Eq. (5). The volume scattering function $\beta$ was measured at $124^{\circ}$ [15] and $660 \mathrm{~nm}$ by a WET Labs ECO BBRT in Exp. 1 and by a WET Labs ECO BB3 in Exp. 2. The beam attenuation coefficient $c$ was measured with an acceptance angle of $0.93^{\circ}$ and a path length of $10 \mathrm{~cm}$ by a WET Labs ac-9 (nine wavelengths between 412 and $715 \mathrm{~nm}$; data reported here are at $650 \mathrm{~nm}$ for comparison with the ECO BB data unless otherwise noted). The LISST also measured $c$, but with a smaller acceptance angle of $0.0269^{\circ}$ and a shorter path length of $5 \mathrm{~cm}$ ( $670 \mathrm{~nm}$ only). The LISST was configured to sample at $0.25 \mathrm{~Hz}$ (Exp. 1) and $0.1 \mathrm{~Hz}$ (Exp. 2), the ac-9 at $6 \mathrm{~Hz}$, and the ECO BB sensors at $1.1 \mathrm{~Hz}$ (Exp. 1) and $1.0 \mathrm{~Hz}$ (Exp. 2). Blank measurements were collected in the sink with both the ac-9 and the ECO BB sensors before addition of the clay. Mean blanks were subtracted from all subsequent measurements to yield $c_{p}$ and the particulate scattering coefficient $\beta_{p}$. The equation $b_{b p}=2 \pi \chi \beta_{p}$ was used to derive $b_{b p}$ from $\beta_{p}$ at $124^{\circ}$, using $\chi=1.08[15]$.

The first $\sim 1.6 \overline{\mathrm{h}}$ of the experiment was dominated by aggregation (referred to hereafter as the "aggregation period"); $\bar{D}_{\text {LISST }}$ increased by a factor of $\sim 10$, accompanied by increased variability in $c_{p}$ and $b_{b p}$ on time scales $<1 \mathrm{~min}$, while mean $c_{p}$ and $b_{b p}$ on scales of minutes changed by less than $25 \%$ (Fig. 1). At $1.6 \mathrm{~h}$ (Fig. 1, gray dashed line), aggregates were recorded in the LISST's largest size bin in significant numbers (10\% of total aggregate area concentration), so after this point some aggregates likely exceed the LISST's upper size limit and are not included in $\bar{D}_{\text {LISST }}$. The remainder of the experiment after $1.6 \mathrm{~h}$ was a period of rapid settling (referred to hereafter as the "settling period"), with decreases in mean $c_{p}$ and $b_{b p}$ exceeding $70 \%$ (Fig. 1 ).

\section{Data Processing for VMR Inversion}

We calculated $\bar{D}_{c p}$ at 3 -min intervals over the course of the experiment using Eq. (2). We similarly calculated $\bar{D}_{b b p}$ by substituting $b_{b p}$ for $c_{p}$ and $Q_{b b}$ for $Q_{c}$ in Eq. (2). In this section we describe in detail the steps taken to calculate $\bar{D}_{c p}$ and $\bar{D}_{b b p}$ from the time series of $c_{p}$ and $b_{b p}$.

\section{Bin Width of VMR Calculation}

The VMR inversion was calculated in 3-min bins to minimize the change in $\bar{D}$ over a single bin while still obtaining an acceptable VMR precision $(<25 \%$ relative standard deviation). If rare particles $\left(E\left[N_{i}(t)\right]<10\right)$ do not dominate the $b_{b p}$ or $c_{p}$ signal, then the VMR precision can be approximated by the precision of the sample variance of a normal distribution [Eq. (7)], where $n_{\mathrm{VMR}}$ is the number of independent samples and Std[VMR] is the standard deviation of the sample VMR [16]. Here $n_{\mathrm{VMR}}$ can be approximated by the ratio of the bin width to $t_{\mathrm{res}}$ or $t_{\mathrm{samp}}$ (whichever is larger) yielding $n_{\mathrm{VMR}}$ of 32-45 for all sensors except the LISST in Exp. 2, whose 10 -s integration time reduced $n_{\mathrm{VMR}}$ to 18 . Theoretical minimum relative VMR precisions calculated via Eq. ( 7 ) and converted to relative $\bar{D}_{c p}$ or $\bar{D}_{b b p}$ precisions are given in Table 3 . In addition to providing sufficient precision, the bin width should also be greater than the filter width (see Subsection 2.C.2) so as not to reduce variance more than intended:

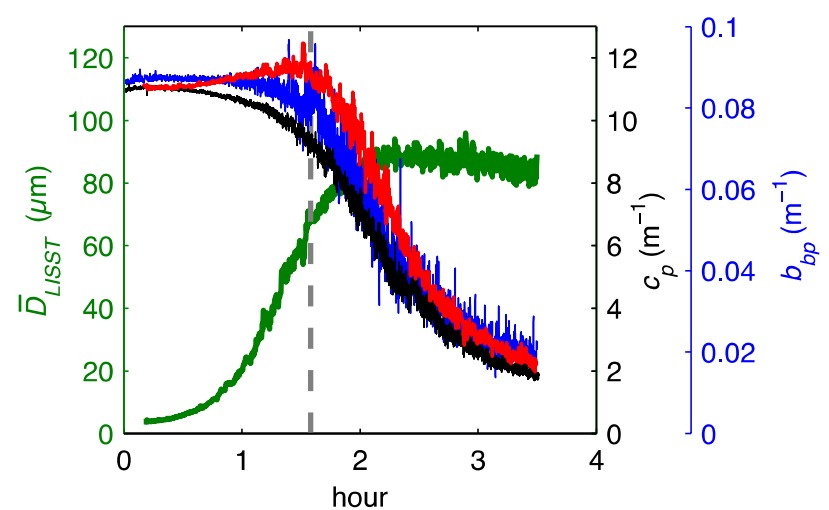

Fig. 1. Time series of unbinned optical data during Exp. 2: $\bar{D}_{\text {LISST }}$ (green), $c_{p}$ from ac-9 (black), and LISST (red), and $b_{b p}$ (blue). Dashed gray line at $1.6 \mathrm{~h}$ divides the "aggregation period" from the "settling period". 
Table 3. VMR Performance During Validation Period $(t=0.75-1.6 \mathrm{~h})$

\begin{tabular}{|c|c|c|c|c|c|c|}
\hline & ECO BBRT (660) & ECO BB3 (660) & ac-9 (650) & ac-9 (650) & LISST (670) & LISST (670) \\
\hline Experiment & Exp. 1 & Exp. 2 & Exp. 1 & Exp. 2 & Exp. 1 & Exp. 2 \\
\hline Mean relative bias (lit. $Q$ ) $\pm 95 \%$ conf. & $38 \pm 10 \%$ & $29 \pm 12 \%$ & $26 \pm 6 \%$ & $20 \pm 6 \%$ & $17 \pm 11 \%$ & $32 \pm 13 \%$ \\
\hline Slope $\pm 95 \%$ conf. (log 10 scale) & $1.04 \pm 0.13$ & $1.16 \pm 0.14$ & $1.02 \pm 0.09$ & $1.02 \pm 0.11$ & $0.97 \pm 0.17$ & $1.07 \pm 0.20$ \\
\hline Relative RMSE & $14 \%$ & $16 \%$ & $10 \%$ & $12 \%$ & $18 \%$ & $24 \%$ \\
\hline$n_{\mathrm{VMR}}$ & 32 & 40 & 42 & 39 & 45 & 18 \\
\hline
\end{tabular}

$$
\frac{\mathrm{Std}[\mathrm{VMR}]}{E[\mathrm{VMR}]}=\sqrt{\frac{2}{n_{\mathrm{VMR}}-1}} .
$$

\section{Detrending Filter}

To minimize variance from sources other than particle size, (e.g., small scale patchiness or nonrandom distribution of particles), $c_{p}$ and $b_{b p}$ time series were detrended by subtracting a filter [e.g., Fig. 2(a), red line] consisting of a running median followed by a running mean, both with windows of $50 \mathrm{~s}$. This filter was chosen after experimentation with various filters on simulated time series. The 50 -s window is approximately 10 times $t_{\text {res }}$ (see Subsection 2.C.3). In simulated time series, this detrending filter with window 10 times either $t_{\text {res }}$ or the sample interval (whichever is larger) preserved $\sim 90 \%$ of the variance due to particle size, while minimizing bias due to any variability in concentration on scales of minutes and longer (data not shown). Note that $t_{\text {res }}$ in this study is calculated using autocorrelations in the detrended data (after applying the filter), so a guess or alternative estimate of $t_{\text {res }}$ must be used when deciding the initial
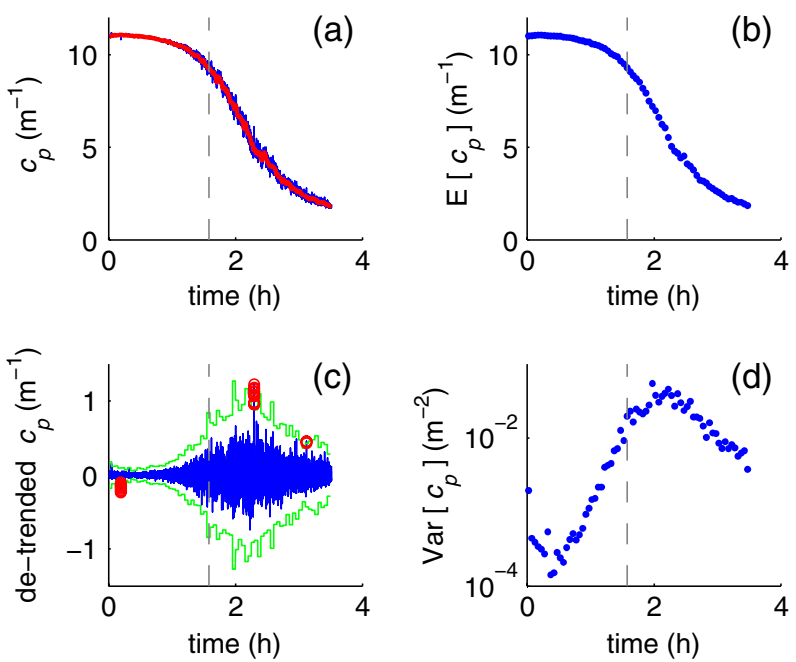

Fig. 2. Calculation of mean and variance of $c_{p}$ from the ac-9 (Exp. 2). (a) Raw unbinned $c_{p}$ time series (blue) and detrending filter (red). (b) Means calculated from raw time series in 3 min bins. (c) Detrended time series (blue), with outlier threshold (green) and outliers (red circles). (d) Variances calculated from detrended time series in 3 min bins. Dashed gray line at $1.6 \mathrm{~h}$ divides the "aggregation" and "settling" periods in all panels. filter width. If the subsequent autocorrelationbased $t_{\text {res }}$ estimates (Subsection 2.C.3) are very different from the initial guess, then a new detrending filter can be applied and a new $t_{\text {res }}$ calculated until the filter width is close to 10 times $t_{\text {res }}$.

\section{Estimation of Residence Time}

To estimate $t_{\text {res }}$, temporal autocorrelations were calculated on the detrended time series at 15 min intervals using Eq. (8), where $\Delta t$ is lag time. Strong positive autocorrelations $(>0.5)$ were found at $\Delta t<2 \mathrm{~s}$ for both ac-9 $c_{p}$ and $b_{b p}$ measurements, and residence time was estimated as twice the lag time corresponding to autocorr $(\Delta t)=0.5$. The median estimated residence times across all 15 min bins for each of the two experiments were 4.3 and $4.6 \mathrm{~s}$ for ac-9 $c_{p}$ and 5.6 and $4.5 \mathrm{~s}$ for $b_{b p}$ (Table 2). We estimate the mean path lengths of particles passing through the sampling volumes of the ECO BB and ac-9 as $1 \mathrm{~cm}$ and $8 \mathrm{~mm}$, respectively, so these residence time estimates are consistent with acoustic Doppler velocimeter measurements (measured in Exp. 2 only) indicating $2-3 \mathrm{~mm} \mathrm{~s}^{-1}$ particle movement throughout the course of the experiment (data not shown). The LISST $c_{p}$ time series were not strongly autocorrelated due to lower sampling frequency, preventing estimation of $t_{\text {res }}$ via Eq. (8). Instead, ac-9 $t_{\text {res }}$ was scaled to the LISST's smaller weighted beam width ( $7 \mathrm{~mm}$ versus $8 \mathrm{~mm}: 0.875 \times$ ) to yield $t_{\text {res }}$ estimates of 3.8 and 4.0 for Exps. 1 and 2, respectively.

$$
\operatorname{autocorr}(\Delta t)=\frac{\sum_{t=1}^{n-L} c_{p}(t) * c_{p}(t+\Delta t)}{\sum_{t=1}^{n-L} c_{p}(t)^{2}} .
$$

\section{Outlier Removal}

To decrease uncertainty in the VMR, large outliers in the detrended time series [Fig. 2(c), red circles] with absolute values greater than a threshold of 4 times the interquartile range of each $3 \mathrm{~min}$ bin [Fig. 2(c), green lines] were eliminated from both the raw and detrended time series $(<0.2 \%$ of data points removed from each time series). Note that very large particles can cause positive outliers, which, if present in sufficient numbers, may be analyzed separately [17-19].

\section{Calculation of Mean and Variance}

After outlier removal, mean $c_{p}$ and $b_{b p}$ were calculated for each bin [Fig. 2(b)] from the unbinned time 
series [Fig. 2(a), blue line]. Variances [Fig. 2(d)] were calculated from the detrended time series [Fig. 2(c), blue line] for the same bins.

\section{Estimation of $Q_{c}$ and $Q_{b b}$}

Empirical estimates of $Q_{c}$ and $Q_{b b}$ over the course of the experiment (Fig. 3) were obtained by dividing mean values of $b_{b p}$ and $c_{p}$ by the total cross-sectional area concentration derived from the LISST PSD [Eq. (6)]. Patterns in $Q_{c}$ and $Q_{b b}$ were similar during both experiments, but $Q_{b b}$ was consistently $\sim 30 \%$ higher during Exp. 2, most likely due to uncertainty in the calibrations of the two ECO BB sensors [20]. Divergence between LISST and ac-9 $Q_{c}$ after the first hour is likely due to the ac-9's larger acceptance angle, which causes it to underestimate somewhat the attenuation due to larger particles [21]. During the settling period the largest aggregates likely surpassed the maximum LISST size bin, leading to an under-estimate of total area concentration and a positive bias in $Q_{c}$ and $Q_{b b}$ estimates. During the aggregation period (Fig. 3, left of vertical dashed lines), changes in estimated $Q_{c}$ and $Q_{b b}$ were small $(<9 \%)$ despite a large increase in $D_{\text {LISST }}$ (Fig. 1), so the median $Q_{c}$ and $Q_{b b}$ from the aggregation period (Table 2) were applied to the entire time series. The use of a single $Q$ also ensures that any trends in $\bar{A}_{c p}$ and $\bar{A}_{b b p}$ are entirely independent from trends in the LISST PSD. Note, however, that the VMR inversion is most valuable in applications where PSD
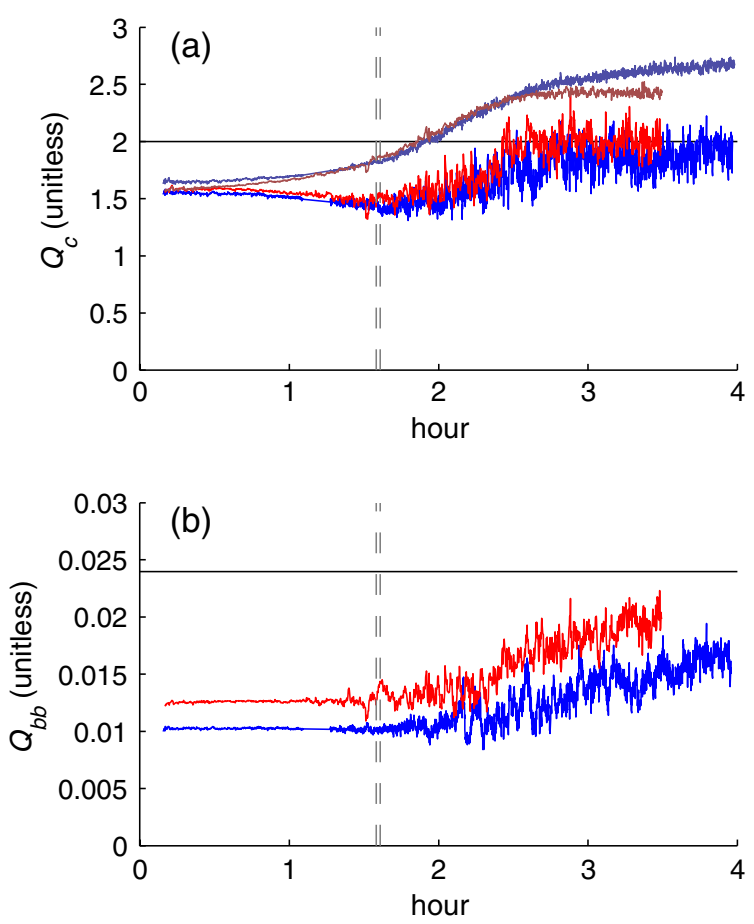

Fig. 3. Time series of direct estimates of $Q_{c}$ from ac-9 $c_{p}$ (panel a; light colors), $Q_{c}$ from LISST $c_{p}$ (panel a; dark colors) and $Q_{b b}$ (panel b) from Exps. 1 (blue) and 2 (red). Horizontal black lines show the literature values of $Q$ used in this study. Dashed gray lines mark the transition from the aggregation period to the rapid settling period. measurements are not available, in which case literature values of $Q_{c}$ and $Q_{b b}$ must be used in lieu of these empirical estimates, adding uncertainty to the VMR inversion.

In order to simulate an application in which $Q_{c}$ could not be determined empirically, we chose the theoretical asymptotic value $Q_{c}=2$ for $D_{i} \gg \lambda$ [22]. $Q_{b b}$ is less well constrained in the ocean, but we can set a likely range of $0.004<b_{b p} / c_{p}<0.035$ based on literature values of marine particulate backscattering ratios $(0.005-0.035)$ [23,24] and single-scattering albedos $(0.8-1.0)$ [25]. In this case, a choice of $Q_{b b}=0.024\left(b_{b p} / c_{p}=0 . \overline{012} ; Q_{c}=2\right)$ constrains additional uncertainty in $Q_{b b}$ within a factor of 3 , equivalent to $\bar{D}_{b b p}$ within a factor of 1.7 (Table 2 ). In this study we evaluate overall VMR inversion performance using these literature values of $Q_{c}$ and $Q_{b b}$ (Figs. 4 and 5), but we use our empirical estimates of $Q_{c}$ and $Q_{b b}$ for the remaining analyses (Figs. $\underline{6}-\underline{8}$ ) in order to explore other possible sources of bias.

\section{Instrument Sampling Volume and Integration} Time, $V$ and $t_{\text {samp }}$

We used an ac-9 sample volume $V$ of $5.0 \mathrm{ml}$ based on manufacturer specifications (beam diameter: $0.8 \mathrm{~cm}$; path length: $10 \mathrm{~cm}$ ). The ECO BB and LISST sample volumes (0.62 and $1.9 \mathrm{ml}$, respectively) were calculated using Eq. (B7) to account for inhomogeneous sensing efficiency within the sample volume (see Appendix B). The ac-9 $t_{\text {samp }}$ at each wavelength is $3 \mathrm{~ms}$ (WET Labs ac-9 User's Guide revision T), and we approximate the ECO BBRT and BB3 $t_{\mathrm{samp}}$ as the inverse of the sampling frequency minus a $50 \mathrm{~ms}$ overhead time: 0.87 and $0.95 \mathrm{~s}$ in Exp. 1 and Exp. 2 , respectively. LISST $t_{\text {samp }}$ is simply the averaging period: 4 and $10 \mathrm{~s}$ in Exps. 1 and 2, respectively. These values of $t_{\mathrm{samp}}$, combined with the values of $t_{\text {res }}$ reported in Subsection 2.C.3, yield correction factors $\alpha(\tau)$ of 0.9998 for the ac-9, and 0.95 and 0.93 for the ECO BBRT and ECO BB3, and 0.65 and 0.35 for the LISST in Exps. 1 and 2, respectively (Table 2).

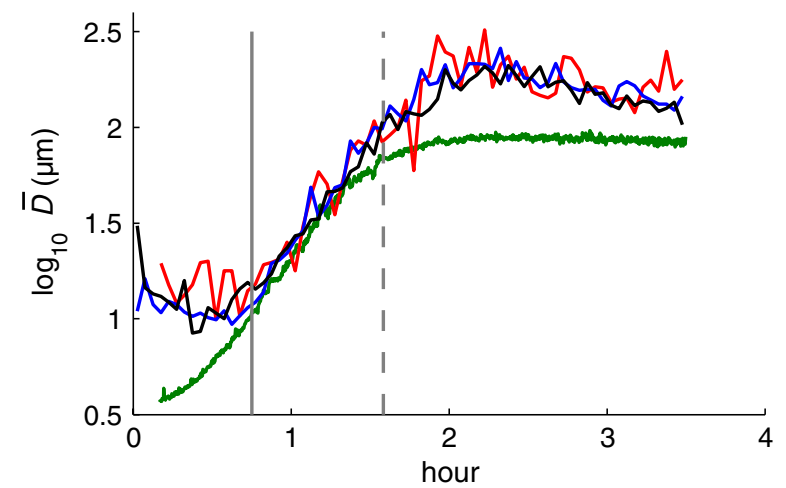

Fig. 4. Time series of $\bar{D}_{\text {LISST }}$ (green), $\bar{D}_{b b p}$ (blue), $\bar{D}_{c p \_a c 9}$ (black), and $\bar{D}_{c p_{\text {LLISST }}}$ (red) during Exp. 2. Literature values of $Q_{b b}$ and $Q_{c}$ were used to calculate $\bar{D}_{b b p}$ and $\bar{D}_{c p}$. Solid gray line marks the start of the validation period. Dashed gray line marks the end of the validation period and the start of the settling period. 

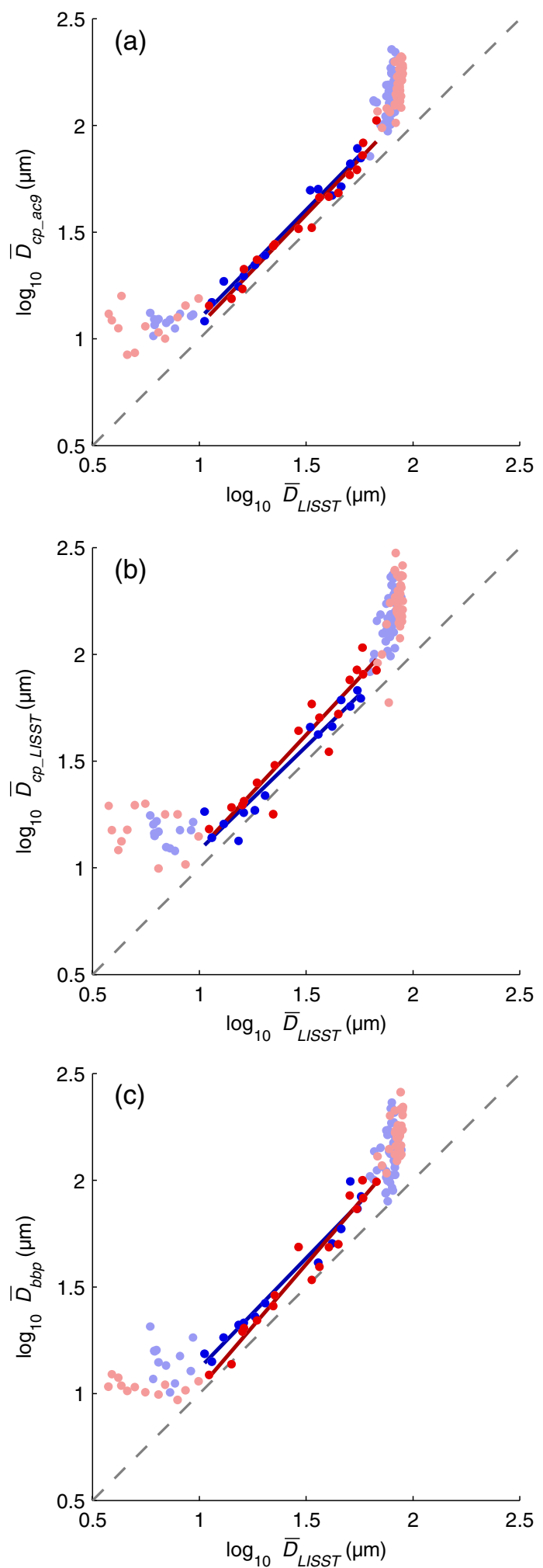

Fig. $\quad 5 . \quad$ (a) $\quad \log _{10}\left(\bar{D}_{\text {LISST }}\right) \quad$ versus $\quad \log _{10}\left(\bar{D}_{c p \_ \text {ac } 9}\right) \quad$ (a), (b) $\log _{10}\left(\bar{D}_{c p \_L I S S T}\right)$ (b), and (c) $\log _{10}\left(\bar{D}_{b b p}\right)$ (from Exps. 1 (blue) and 2 (red). Literature values of $Q_{b b p}$ and $Q_{c}$ were used to calculate $\bar{D}_{b b p}$ and $\bar{D}_{c p}$. Solid lines are type-I linear regressions of logtransformed data. Pale data points, from outside the validation period, are excluded from the regressions. Slopes ( $\pm 95 \%$ conf.) and $r^{2}$ values of are given in the Table 3 , along with the mean biases ( $\pm 95 \%$ conf.).

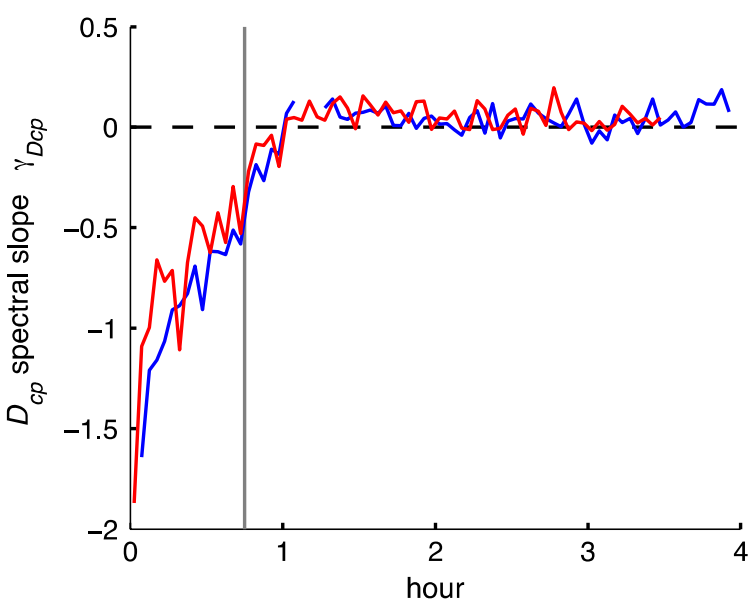

Fig. 6. Spectral slope $\gamma_{D c p}$ of $\bar{D}_{c p}(\lambda)$ over the course of Exps. 1 (blue) and 2 (red). Empirical estimates of $Q$ were used at each wavelength to calculate $\bar{D}_{c p}$. Horizontal dashed line denotes no spectral dependence, and vertical gray line indicates the start of the validation period.

\section{Results}

\section{A. VMR Inversion and Comparison with LISST}

Patterns of $\bar{D}_{c p}$ (Fig. $\underline{4}$, black and red lines) and $\bar{D}_{b b p}$ (Fig. 4, blue line) were similar. At the start of both experiments, there was a factor of $\sim 2.5$ discrepancy between $\bar{D}_{\text {LISST }}$ and both $\bar{D}_{c p}$ and $\bar{D}_{b b p}$. This discrepancy disappeared gradually over the first $0.75 \mathrm{~h}$. This "period of initial bias" is discussed in Subsection 4.A.2 and is excluded from the validation regressions in Fig. 5. During the "validation period" from hour 0.75 (Fig. 4, solid gray line) to the start of the settling period (Fig. $\underline{4}$, dashed gray line), $\bar{D}_{\text {LISST }}$ agreed closely with both $\bar{D}_{b b p}$ and $\bar{D}_{c p}$. Validation regressions of both $\log _{10}\left(\bar{D}_{b b p}\right)$ and $\log _{10}\left(\bar{D}_{c p}\right)$ against $\log _{10}\left(\bar{D}_{\text {LISST }}\right)$ from this period (Fig. 5, bold colors) were highly linear $\left(r^{2} \geq 0.95\right)$ and slopes were close to $1: 1$. Mean relative biases of $\bar{D}_{c p}$ and $\bar{D}_{b b p}$ ranged from $+17 \%$ to $+38 \%$ (Table 3). When literature $Q_{c}$ and $Q_{b b}$ were replaced by empirical estimates (Table 2), relative biases decreased to $-11 \%-20 \%$ (Table 3 ). During the settling period beginning at $1.6 \mathrm{~h}$ (dashed gray lines in Fig. $\underline{4}$ ), $\bar{D}_{b b p}$ and $\bar{D}_{c p}$ continued to increase to a peak of approximately $200 \mu \mathrm{m}$ at $2.2 \mathrm{~h}$ and then declined to $\sim 100 \mu \mathrm{m}$ by the end of the experiment (Fig. 4), presumably as the largest aggregates settled out of suspension. $\bar{D}_{\text {LISST }}$ remained lower and more constant during the settling period, never exceeding $100 \mu \mathrm{m}$, likely due the LISST's inability to detect particle sizes $>230 \mu \mathrm{m}$. During the settling period, the LISST area PSD was sharply peaked at the largest size bin, strongly suggesting the presence of larger aggregates, but a "stranded" population of particles $<10 \mu \mathrm{m}$ maintained $\bar{D}_{\text {LISST }}$ below $100 \mu \mathrm{m}$ [14].

\section{B. Spectral Dependence of $\bar{D}_{c p}$}

A power law of the form $c_{p}(\lambda) \sim \lambda^{-\gamma_{c p}}$ (where $\lambda$ is wavelength) was fit to the ac-9 $c_{p}$ time series, and the 
resulting spectral slope $\gamma_{c p}$ varied between 1.35 and 1.6 during the experiments [14]. Both $E\left[c_{p}\right]$ and the empirical $Q_{c}$ (calculated separately at each wavelength) have the same spectral slope: $\gamma_{c p}$. If the VMR inversion assumptions hold, the spectral slope of $\operatorname{Var}\left[c_{p}\right]$ should be twice $\gamma_{c p}$. These spectral dependencies should cancel out in Eq. (2), so $\gamma_{D c p}$, the spectral slope of $\bar{D}_{c p}$, should be zero (i.e., $\bar{D}_{c p}$ is accurate at all wavelengths), regardless of $\gamma_{c p}$. However, during the first $0.75 \mathrm{~h}$ of the experiment, coincident with the disagreement between $\bar{D}_{c p}$ and $\bar{D}_{\text {LISST }}$, we observed a strong spectral dependence of $\bar{D}_{c p}$ (Fig. 6 ). The $\bar{D}_{c p}$ spectral slope was steepest $\left(\gamma_{D c p}<-1 . \overline{5}\right)$ at the start of the experiments, increased to zero (no spectral dependence) in the first hour, and remained within $0 \pm 0.2$ for the remainder of the experiments (Fig. 6). The low $\gamma_{D c p}$ during the first 0.75 indicates a violation of at least one VMR inversion assumptions and provides further justification for rejecting the early VMR inversion results. We discuss possible sources of both the low $\gamma_{D c p}$ and the early disagreement between $\bar{D}_{c p}$ and $\bar{D}_{\text {LISST }}$ in Subsection 4.A.2.

\section{Relationship Between $\bar{D}$ and Clearance Rate $R_{c p}$}

The rate at which water at the depth of the sensors is cleared of particles is a function of sinking rate (as well as vertical concentration gradient and convective mixing rate). The rate of relative decrease in $c_{p}$ per minute, $R_{c p}$ [Eq. (9)] is a measure of particle clearance rate. Because particle size is a primary driver of sinking rate [26], we expect that $R_{c p}$ will be positively related to $\overline{\bar{D}_{c p}}$; Fig. $7($ a) shows that it is. The $c_{p}$ time series was smoothed using a secondorder polynomial spline with a 70 min window before the calculation of $R_{c p}$. The first derivative of the smoothed $c_{p}$ time series was approximated at time $t$ (in min) using a 6 min window and normalized to $c_{p}$ to yield $R_{c p}$ [Eq. (9)]:

$$
R_{c p}=-\frac{1}{c_{p}} \frac{d c_{p}}{d t} \approx \frac{1}{c_{p}} \frac{c_{p}(t-3 \mathrm{~min})-c_{p}(t+3 \mathrm{~min})}{6 \mathrm{~min}} .
$$

$\bar{D}_{c p}$ was further averaged into 9-min bins to reduce variability. There was a strong positive relationship between $R_{c p \_a c 9}$ and all estimates of $\bar{D}$ during the aggregation phase [Fig. 7(a), plus sign]. During the settling phase [Fig. 7(a), open circles], the relationships of $R_{c p_{-} \text {ac9 } 9}$ with $\bar{D}_{c p_{\_} \text {ac } 9}$ and $\bar{D}_{b b p}$ change little while the relationship with $\bar{D}_{\text {LISST }}$ changes sharply. Figure 7 (a) supports the idea that the LISST PSD misses large particles during the settling period and provides qualitative support for the validity of $\bar{D}_{c p}$ when the LISST could not be used for quantitative validation. For comparison, the spectral slopes of $c_{p}$ and $b_{b p}$, two other proposed proxies for particle size [27,28], did not maintain strong, consistent relationships between either $\bar{D}_{\text {LISST }}$ [14] or $R_{c p \_a c 9}$ [Fig. $\underline{7(\mathrm{~b})]}$ during
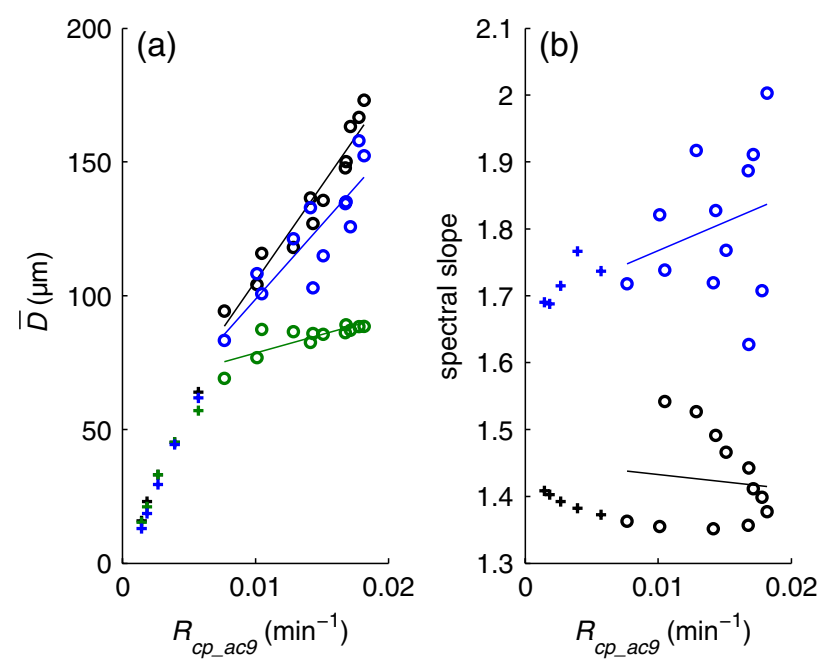

Fig. 7. (a) Relationships between $R_{c p_{-} \text {ac } 9}$ and $\bar{D}_{c p_{-} \text {ac9 }}$ (black), $\bar{D}_{b b p}$ (blue), or $\bar{D}_{\text {LISST }}$ (green). (b) Relationships between $R_{c p \_ \text {ac9 }}$ and spectral slopes of $c_{p}$ (black) and $b_{b p}$ (blue). Data points in both panels are $9 \mathrm{~min}$ bin averages from the validation period (+) and settling period (o) of Exp. 2. Solid lines show type I linear regressions of $R_{c p \_ \text {ac9 }}$ against settling period $\bar{D}_{c p \_ \text {ac } 9}\left(r^{2}=0.93\right), \bar{D}_{b b p}$ $\left(r^{2}=0.75\right), \bar{D}_{\text {LISST }}\left(r^{2}=0.63\right), c_{p}$ spectral slope $\left(r^{2}=0.01\right)$, and $b_{b p}$ spectral slope $\left(r^{2}=0.07\right)$. Regressions in panel (a) are all highly significant $(p<0.01)$, whereas regressions in panel (b) are not $(p>0.4)$. Empirical estimates of $Q_{c p}$ and $Q_{b b p}$ were used to calculate $\bar{D}_{c p \_a c 9}$ and $\bar{D}_{b b p}$.

this experiment. The relationship between $R_{c p}$ and $\bar{D}$ is discussed further in Subsection 4.A.3.

\section{Comparison with Shifrin's Inversion}

The Shifrin inversion is given in Eq. (10), adapted from Shifrin [13] (his Eq. 6.28), where $T$ is the transmittance, defined as the ratio of transmitted light in the presence of particles to transmitted light in the absence of particles, $C$ is the attenuance, defined as $\ln \left(T^{-1}\right), S$ is the cross section of the transmissometer beam, and $\Phi$ is a function of $C$, given in Eq. (11) (adapted from Shifrin [13]; his Eqs. 6.18, 6.21) and $\psi$ is an integration variable in Eq. (11):

$$
\begin{gathered}
\bar{A}_{\text {Shifrin }}=\frac{E[C(t)] \operatorname{Var}[T(t)]}{\phi(E[C(t)])} \frac{S}{Q_{c}}, \\
\phi(C)=2 C e^{-2 C} \int_{0}^{\pi}\left[\exp \left(\frac{C(\psi-\sin \psi)}{\pi}\right)-1\right] \sin \psi \mathrm{d} \psi .
\end{gathered}
$$

Note that Shifrin's [13] Eq. 6.18 contains a misprint, but the correct equation is in an earlier paper [12] (his Eq. 3).

As with the VMR inversion, we detrended $T$ and removed outliers before calculating the variance and calculated the Shifrin inversion for $3 \mathrm{~min}$ bins. The integral in Eq. (11) was numerically approximated using a quadrature algorithm (MATLAB, "integral.m"). As before, $\bar{A}_{\text {Shifrin }}$ was converted to $\bar{D}_{\text {Shifrin }}$ using Eq. (). 
The magnitude and patterns in $\bar{D}_{\text {Shifrin }}$ and $\bar{D}_{c p}$ were very similar, including the initial bias and the disagreement with $\bar{D}_{\text {LISST }}$ during the settling period, but the two methods diverged at higher values of $c_{p}$. Both $\bar{D}_{\text {Shifrin }}$ and $\bar{D}_{c p}$ were calculated from all ac-9 wavelengths during the validation period using wavelength-specific empirical $Q_{c}$ estimates and divided by corresponding values of $\bar{D}_{\text {LISST }}$ to estimate bias. Mean relative biases were 1.09 for $\bar{D}_{c p}$ and 0.91 for $\bar{D}_{\text {Shifrin. }}$ Type I linear regressions against $C$ show a significant negative correlation between $C$ and bias in $\bar{D}_{\text {Shifrin }}$ but not between $C$ and $\bar{D}_{c p}$ bias, causing the two inversions to converge as $C$ approached zero (Fig. 8). This result is discussed in Subsection 4.C.

\section{Discussion}

\section{A. VMR Accuracy}

The strong agreement between $\bar{D}_{b b p}, \bar{D}_{c p_{\_} \text {ac } 9}$, and $\bar{D}_{c p \_ \text {LISST }}$ throughout the experiment (Fig. $\underline{4}$ ) indicates that the VMR inversion is reliable and robust to differing principles of instrument operation, differing sampling volumes, differing bin averaging, and even differing transmissometer acceptance angles (even though the larger acceptance angle of the ac9 causes it to underestimate $c_{p}$ due to the larger particles [14]). However, the VMR inversion does not always agree with $\bar{D}_{\text {LISST }}$. We asses the accuracy of the VMR inversion (and the LISST) during three distinct periods.

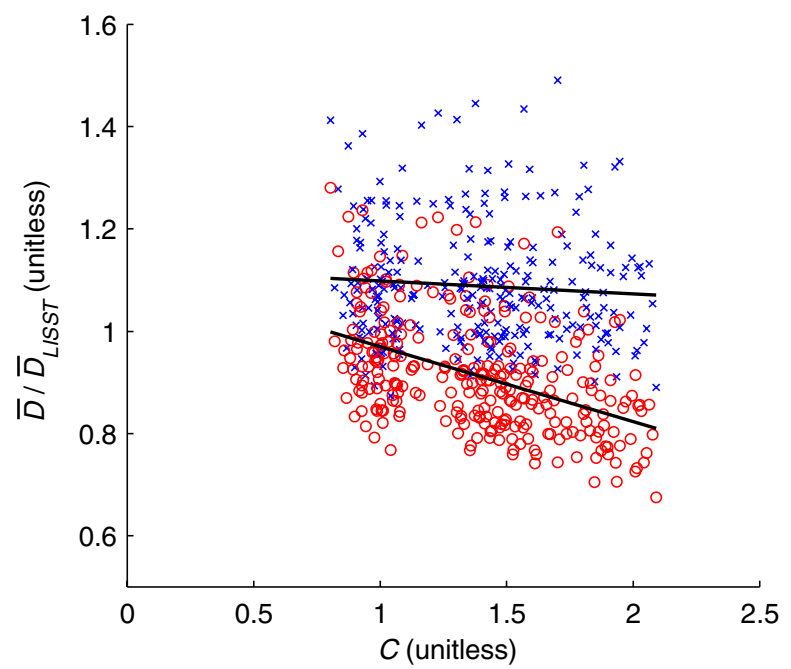

Fig. 8. Bias in $\bar{D}_{c p}$ relative to the $\bar{D}_{\text {LISST }}$ (blue x's) is not significantly correlated with attenuance $C$ (type I linear regression: $y=-0.02 \pm 0.04 x+1.12 \pm 0.06 ; \quad r^{2}=0.01$ ). Relative bias in $\bar{D}_{\text {Shifrin }}$ (red o's) decreases as a function of $C$ (type I linear regression: $\left.y=-0.15 \pm 0.03 x+1.12 \pm 0.05 ; r^{2}=0.21\right)$. Data from the validation period of both experiments at all ac-9 wavelengths are included. Empirical estimates of $Q_{c p}$ and $Q_{b b}$ were used at each wavelength to calculate both $\bar{D}_{c p}$ and $\bar{D}_{\text {Shifrin }}$.

\section{Validation Period}

During the validation period, $\bar{D}_{c p}$ and $\bar{D}_{b b p}$ were strongly correlated with $\bar{D}_{\text {LISST }}$ over nearly an order of magnitude (10-80 $\mu \mathrm{m} ; r^{2} \geq 0.95-\log$ scale) with slopes close to $1: 1$ and mean biases of $17 \%-38 \%$ (Fig. 5; Table 3). The largest single cause of bias is uncertainty in $Q_{b b}$ and $Q_{c}$; use of direct estimates (Table 2) reduced biases to $-11 \%$ to $+20 \%$. The causes of these remaining small but significant mean biases are unknown, but may include uncertainty in $V$, slightly nonrandom particle distribution, a small size-dependence of $Q_{b b}$ and $Q_{c}$, instrument noise proportional to the signal, and/or a slight low bias in $\bar{D}_{\text {LISST }}$ due to particles $>230 \mu \mathrm{m}$. Given the uncertainties involved in estimating particle size by any method, these results are encouraging. For comparison, mean discrepancies of $11 \%$ were found between median particle diameters measured by a LISST and a Coulter counter across a wide variety of particle types [29]. Biases of up to $38 \%$ may often be acceptable in natural systems where $\bar{D}$ varies by a factor of 2 or more. Furthermore, the strong relative agreement between $\bar{D}_{c p}, \bar{D}_{b b p}$, and $\bar{D}_{\text {LISST }}$ (regression slopes on log scale: 1.02-1.16) supports the use of the VMR inversion as a relative size indicator, regardless of the choice of $Q_{b b}$ or $Q_{c}$.

\section{Initial Bias}

The assumption that a single value of $Q_{b b}$ or $Q_{c}$ applies to all particles may introduce some bias in the VMR inversion, but it is unlikely that variability in efficiencies can explain the large (factor of 6) and ephemeral bias, observed at the start of the experiments, given the stability of $Q_{c}$ and $Q_{b b}$ estimates throughout the aggregation phase (Fig. 3 ), indicating minimal relationship between $Q_{b b}$ or $\overline{Q_{c}}$ and size.

The discrepancy between $\bar{D}_{\text {LISST }}$ and both $\bar{D}_{c p}$ and $\bar{D}_{b b p}$ during the first $0.75 \mathrm{~h}$ is most likely caused by an additional source of variance other than particle size, leading to a positive bias in the VMR inversion. This extra variance apparently decreased over most of this early period [e.g., Fig. 2(d)], causing corresponding decreases in $\bar{D}_{c p}$ and $\overline{\bar{D}_{b b p}}$ (despite increasing particle size). The strong negative $\gamma_{D c p}$ associated with this extra variance indicates that its spectral slope is likely flat or even negative (higher at higher wavelengths and opposite to $\gamma_{c p}$ ). The cause of this extra variance is not clear. Nonrandom (patchy) distribution of particles at the start of the experiment would increase variance. However, such clustering would cause the same relative bias at all wavelengths and cannot explain the negative $\gamma_{D c p}$ (Fig. $\underline{6}$ ). The initial mixing of salt into the sink would have caused inhomogeneity in index of refraction ("schlieren"), which could lead to fluctuations in $c_{p}$, but this effect would also increase mean $c_{p}$ and interfere with the LISST PSD inversion [30]. The source of the variance could be electronic noise, but it is unclear why it 
would affect all sensors in both experiments in such similar ways.

Regardless of its source, the relative effect of added variance is greatest at low $\bar{D}$, when variance due to particle size is small; after $0.75 \mathrm{~h}$, the rapid increase in variance due to increasing $\bar{D}$ likely overwhelmed any remaining bias.

\section{3. $\quad$ Settling Period}

During the settling period, the presence of aggregates too large for the LISST inversion to constrain prevented quantitative assessment of VMR inversion accuracy. However, the strong positive relationships between $\bar{D}_{c p}, \bar{D}_{b b p}$, and $R_{c p}$ during this period [Fig. 7(a)] provide qualitative validation that $\bar{D}_{c p}$ and $\bar{D}_{b b p}$ continue to track particle size. Perhaps more importantly, this relationship demonstrates the potential of the VMR inversion as a tool for studying the dynamics of particle settling in the ocean.

\section{B. VMR Precision}

The $\bar{D}_{\text {LISST }}$ time series was much smoother than the $\bar{D}_{b b p}$ and $\bar{D}_{c p}$ time series (Fig. $\underline{4}$ ), despite much shorter averaging times (10 s versus $3 \mathrm{~min}$ ), suggesting much higher $\bar{D}_{\text {LISST }}$ precision. The root mean square errors (RMSEs) of the validation regressions should therefore be close to the theoretical precision of the VMR, plus the effect of any nonlinearity in the regressions. The RMSEs of the $\bar{D}_{c p_{-} \text {ac } 9}$ validation regressions correspond to relative precisions of $10 \%$ and $12 \%$, close to the theoretical relative precision of $11 \%$ (Table 3 ). $\bar{D}_{c p \_ \text {LISST }}$ and $\bar{D}_{b b p}$ relative RMSEs were higher than theoretical values by $2-8$ percentage points, perhaps due to added uncertainty from inhomogeneous sample volumes. These results support the use of Eq. (7) to provide an initial estimate of VMR precision for planning sampling strategy, although sensor-specific details may decrease precision somewhat, in which case wider bins may be needed to achieve target precision.

\section{Comparison with Shifrin}

The performance of the Shifrin inversion was similar to that of the VMR inversion; agreement between the two was usually within $20 \%$ (Fig. 8 ) and improved at longer wavelengths and lower $C$. However, the disagreement at high values of $C$ is a result of differing inversion assumptions, which are discussed in this section.

While the VMR inversion attributes $\operatorname{Var}\left[c_{p}\right]$ entirely to random fluctuations in the number of particles in $V$, the Shifrin inversion includes an additional source of variance: fluctuations in the degree of particle-particle shading in the beam (see Appendix D). Consequently, Shifrin predicts a higher $\operatorname{Var}\left[c_{p}\right]$ for a given $\bar{D}$ and $E\left[c_{p}\right]$, or a lower $\bar{D}$ for a given $\operatorname{Var}\left[c_{p}\right]$ and $E\left[c_{p}\right]$. The difference between $\bar{D}_{c p}$ and $\bar{D}_{\text {Shifrin }}$ is primarily a function of attenuance $C$, because at higher $C$, particle-particle shading is greater and fluctuations in shading have a larger potential effect on $\operatorname{Var}\left[c_{p}\right]$. If variable shading indeed contributes to measured $\operatorname{Var}\left[c_{p}\right]$, we expect $\bar{D}_{\text {Shifrin }}$ to be more robust than $\bar{D}_{c p}$ to changes in $C$. Instead, we found that $C$ was significantly correlated with bias in $\bar{D}_{\text {Shifrin }}$ but not $\bar{D}_{c p}$ (Fig. $\underline{8}$ ).

This result suggests that the effect on $\operatorname{Var}\left[c_{p}\right]$ of variations in shading within the sample beam was significantly smaller than that predicted by Shifrin. The irregular, elongated shapes [14] and possible semi-transparency of the aggregates in this study may account for some of the reduction in variability versus Shifrin's assumption of solid, circular particle cross sections. Additionally, the fluctuations that were produced by shading, especially of the smallest particles, may have occurred at frequencies too high for the ac-9 to resolve. Therefore, while the VMR inversion appears to be a more consistent method for estimating $\bar{D}$ from $c_{p}$ fluctuations in this experiment, the Shifrin inversion may perform better in conditions of more solid particles, slower relative particle speed, or shorter sample integration times. High variance on time scales much shorter than $t_{\mathrm{res}}$ is expected in such cases, however, and the VMR inversion may still be used if this higher-frequency variance is identified and filtered out.

\section{Comparison with Spectral Slope}

The spectral slopes of $c_{p}$ and $b_{b p}$ have been proposed as in situ optical proxies for particle size [27,28]. Spectral slopes provide certain advantages over the VMR method: no single $Q_{c}$ must be assumed, $\gamma$ can be calculated over shorter temporal and spatial windows than the VMR, $\gamma$ is not biased by noise or other variance, and ocean color measurements may allow estimation of $\gamma_{b b p}$ from space $[28,31]$. On the other hand, $\gamma$ requires more complex, multiwavelength sensors and conversion of $\gamma$ to particle size requires assumptions of Jungian PSD shape and homogeneous spherical particles, often violated in natural particle assemblages. During this study, the VMR inversion performed qualitatively better during the settling period and as a whole over the entire experiment, whereas $\gamma_{c p}$ (although not $\gamma_{b b p}$ ) performed qualitatively better during the initial $0.75 \mathrm{~h}$. Ultimately, our results show that each method can be sensitive to factors other than mean particle size, and more work needs to be done to understand and quantify these other effects. Then, the combination of both methods may provide enhanced information about different parts of the PSD or perhaps about particle composition.

\section{E. Considerations for Field Deployment}

\section{Choice of $Q$}

The literature values of $Q_{c}$ and $Q_{b b}$ presented in this study (Table 2) are intended as first guesses to apply to particles of unknown composition sampled using visible wavelengths. If local particle optical properties are better constrained, site-specific values of $Q$ 
may increase VMR inversion accuracy. Alternatively, if the ratio of $c_{p}$ or $b_{b p}$ to another physical particle measurement (e.g., suspended particulate mass or particulate organic carbon concentration) is approximately constant, this ratio may be substituted for $Q$ in Eq. (2), yielding an estimate of weighted mean particle mass, for example, in place of $A$.

It is important to note that even if bulk $Q$ is well constrained, a strong size dependence of $Q$ within a single sample violates an assumption of the VMR inversion. In this case, it is more accurate to use Eq. (A11), yielding $\bar{A}^{*}$, the mean $A_{i}$ weighted by optical cross section and not physical cross section (see Appendix A). $\bar{A}^{*}$ still provides useful bulk particle size information, but it is more difficult to validate using PSD measurements and may not correlate as strongly with particle clearance rate. In this study, neither $Q_{c}$ nor $Q_{b b}$ varied strongly with size, so both $\bar{A}_{c p}$ and $\bar{A}_{b b p}$, estimates were accurate within $40 \%$ or better. Some work to date has shown that $Q_{c}$ appears well constrained in situ, in accordance with theory [32], but $Q_{b b}$ is likely more variable, especially for mixed populations of mineral and organic particles. Ultimately, in situ field studies are needed to test the accuracy of the VMR inversion in natural particle populations.

\section{Uncertainty in $t_{\text {res }}$}

In sampling regimes in which $t_{\text {res }}$ is smaller than the interval between consecutive samples (as with the LISST in Exp. 2), temporal autocorrelation will be minimal, preventing the estimation of $t_{\text {res }}$ via autocorrelation (Subsection 2.C.3). If possible, therefore, sampling frequency should be increased or platform speed decreased until a temporal autocorrelation is observed. If this is not possible, but the velocity of the sample relative to the sensor is known, $t_{\text {res }}$ may be calculated based on this velocity and sensor geometry. Otherwise, uncertainty in $t_{\text {res }}$ will propagate to uncertainty in $\bar{A}_{b b p}$ or $\bar{A}_{c p}$. However, as long as $t_{\text {res }}$ is constant, VMR inversion relative size estimates will still be useful.

\section{Uncertainty Due to $n_{V M R}$}

In order to apply the VMR inversion to vertical profile data containing strong gradients in $c_{p}$ and $b_{b p}$, profiling speed should be slow enough and sampling frequency high enough that $n_{\mathrm{VMR}} \geq 30$ within a layer of interest to achieve a theoretical relative VMR precision of $\leq 25 \%$ via Eq. (7). Alternatively, the results of multiple profiles through the same water mass may be combined to increase precision.

\section{Unconstrained Variance and the Limits of Detectable Size}

The dimensions of $V$ set a hard upper limit on the size of particles detectable by the VMR inversion. Particles whose diameters approach (or exceed) the smallest dimension of $V$ will often (or always) occur only partially within $V$, "appearing" as smaller particles to the VMR inversion. In practice, however, the largest size of particle included in the VMR inversion may be set by the likelihood of encountering a particle of a given size in $V$ during the course of a single VMR calculation. In this case, large particles too rare to be sampled reliably should be explicitly excluded using an outlier filter, as explained in Subsection 2.C.4.

The lower limit of the size of particles that contribute to scattering (and therefore to VMR inversion estimates of $\bar{D}$ ) is set by the relationship between $D_{i}$ and $Q_{c}$ (or $Q_{b b}$ ). In general, $Q$ decreases with decreasing $D_{i}$ for $D_{i}<\lambda$ (i.e., $D_{i}<0.5 \mu \mathrm{m}$ for visible wavelengths), although the precise lower size limit will also depend on particle shape and index of refraction [33]. In addition, for any given mean signal, there will be a lower limit of detectable $\bar{D}$, set by the variance due to sources other than particle size (e.g., patchy distribution of particles, schlieren [30], or instrument noise). Such "extra" variance, along with uncertainty in $Q_{b b}$, has the potential to cause significant bias in field application of the VMR inversion. During the first $0.75 \mathrm{~h}$ of this experiment, unconstrained noise set the lower limit of detectable $\bar{D}$ at $\sim 10 \mu \mathrm{m}$. Further studies will be necessary to better understand and constrain potential sources of variance and determine this lower limit in field applications. If variance from a particular source is independent of size and well characterized, it can be subtracted from $\operatorname{Var}\left[c_{p}\right]$ or $\operatorname{Var}\left[b_{b p}\right]$ before calculating the VMR inversion, lowering the limit of detectable $\bar{D}$.

\section{Optimal Sample Volume}

In general, larger sample volumes will be more effective at capturing larger particles and smaller sample volumes will be better at distinguishing smaller sizes. An ideal $V$ would therefore be just large enough to reliably capture the largest particles of interest, both physically and statistically (see previous section). In some cases, a pair of sensors with different sample volumes may yield extra information about the PSD, as the smaller volume will exclude the larger particles and be more sensitive to smaller particles. However, other factors, such as sensor sensitivity and signal-to-noise ratio, sample duration, internal averaging and platform speed may be just as important as $V$ for determining sensitivity to small particles and should all be taken into account when choosing an appropriate sampling strategy for use with the VMR inversion.

\section{Conclusions}

We find that the VMR inversion consistently produced accurate estimates of $\bar{D}$ in the range of 10-80 $\mu \mathrm{m}$ during two laboratory clay aggregation experiments. At higher sizes (up to $200 \mu \mathrm{m}$ ), the VMR inversion yielded plausible results that retained the expected correlation with clearance rates, but these higher estimates could not be quantitatively 
validated because the larger aggregates likely exceeded the size range of the LISST inversion. For $\bar{D}<10 \mu \mathrm{m}$, VMR estimates were biased high, likely due to an additional unconstrained source of variance at the start of the experiment.

Testing the VMR inversion in situ will be important to determine the effect of the added complexity of marine environments on inversion accuracy. However, in light of the large range of particle sizes that contribute to optical backscattering and beam attenuation in the ocean, from submicrometer bacteria to aggregates of several millimeters or greater, we believe that the VMR inversion is likely to provide useful mean particle size information in a variety of marine environments, even where sources of bias exist. If so, this inversion, combined with the widespread current and future deployment of small optical sensors, promises to greatly expand the spatial and temporal coverage of marine particle size estimates at little extra cost, leading to increased understanding of particle size in the ocean and the impact of size on the many processes that particles mediate.

Inversion development and analysis were funded by a NASA fellowship (grant NNX-10AP29H to Briggs), the NASA Ocean Biology and Biogeochemistry (OBB) program (grant NNX-13AC42G), and the National Science Foundation (grants OCE-0628107 and OCE-0628379); laboratory experiments were funded by the Office of Naval Research (contract N00014-10-1-0508). The authors thank the two reviewers, Michael Twardowski and Giorgio Dall'Olmo, for their thorough and insightful comments, which greatly improved this manuscript. Thank you also to George Jackson and Ivona Cetinić for helpful discussions and comments and to Clementina Russo and Jim Loftin for help with the laboratory experiments.

\section{Appendix A: Derivation for an Ideal Sensor}

The VMR inversion can be applied to any additive optical property. Here, as in Section $\underline{2}, c_{p}$ is used as an example. The VMR inversion rests on the assumption that particles are randomly distributed in space. For the derivation in this section, let us also assume an ideal sensor, which can measure instantaneously the particulate attenuation $c_{p}(t)$ at time $t$ within the sample volume $V$. Let $c_{p, i}(t)$ be the portion of $c_{p}(t)$ due to a single class $i$ of identical particles. Equation (A1) defines $c_{p, i}(t)$ [22] as a function of $V$, the number of particles $N_{i}(t)$ of class $i$ within $V$, and the attenuation cross-section $\sigma_{c, i}$ of a single particle:

$$
c_{p, i}(t)=N_{i}(t) \frac{\sigma_{c, i}}{V}
$$

In the case of multiple particle classes, $c_{p}(t)$ is simply the sum of $c_{p, i}(t)$ from all particle classes:

$$
c_{p}(t)=\sum_{i} c_{p, i}(t)=\frac{1}{V} \sum_{i} N_{i}(t) \sigma_{c, i}
$$

Similarly, the expected value (mean) of $c_{p}(t)$, $E\left[c_{p}(t)\right]$, can be expressed in terms of $E\left[N_{i}(t)\right]$ :

$$
E\left[c_{p}(t)\right]=E\left[\frac{1}{V} \sum_{i} N_{i}(t) \sigma_{c, i}\right]=\frac{1}{V} \sum_{i} E\left[N_{i}(t)\right] \sigma_{c, i} .
$$

The equality $\operatorname{Var}[a X]=a^{2} \operatorname{Var}[X]$ (where $a$ is constant), and the equality $\operatorname{Var}[X+Y]=\operatorname{Var}[X]+$ $\operatorname{Var}[Y]$ (where $X$ and $Y$ are independent, random variables) allow us to similarly express the variance $\operatorname{Var}\left[c_{p}(t)\right]$ in terms of $\operatorname{Var}\left[N_{i}(t)\right]$ :

$$
\begin{aligned}
\operatorname{Var}\left[c_{p}(t)\right] & =\operatorname{Var}\left[\frac{1}{V} \sum_{i} N_{i}(t) \sigma_{c, i}\right] \\
& =\left(\frac{1}{V}\right)^{2} \sum_{i} \operatorname{Var}\left[N_{i}(t)\right] \sigma_{c, i}^{2} .
\end{aligned}
$$

The assumption that particles are randomly distributed in space allows us to approximate $N_{i}(t)$ as a Poisson random variable, in which case

$$
\operatorname{Var}\left[N_{i}(t)\right]=E\left[N_{i}(t)\right]
$$

After substituting Eq. (5) into Eq. (4), the VMR becomes

$$
\frac{\operatorname{Var}\left[c_{p}(t)\right]}{E\left[c_{p}(t)\right]}=\frac{\bar{\sigma}_{c}}{V},
$$

where $\bar{\sigma}_{c}$ is the mean $\sigma_{c, i}$, weighted by the total attenuation cross-section $\sum_{i} E\left[N_{i}(t)\right] \sigma_{c, i}$ of each class:

$$
\bar{\sigma}_{c}=\frac{\sum_{i} E\left[N_{i}(t)\right] \sigma_{c, i}^{2}}{\sum_{i} E\left[N_{i}(t)\right] \sigma_{c, i}} .
$$

Particle cross-sectional area $A_{p, i}$ can be related to $\sigma_{c, i}$ through the attenuation efficiency factor $Q_{c, i}$, defined as the fraction of light incident on a particle that is attenuated:

$$
Q_{c, i}=\frac{\sigma_{c, i}}{A_{p, i}}
$$

In the event that $Q_{c, i}$ is constant across all particle classes in suspension, we can rewrite Eq. (A7) as

$$
\bar{\sigma}_{c}=\frac{Q_{c}^{2}}{Q_{c}} \frac{\sum_{i} E\left[N_{i}(t)\right] A_{i}^{2}}{\sum_{i} E\left[N_{i}(t)\right] A_{i}}=Q_{c} \bar{A},
$$

where $\bar{A}$ is the mean cross-sectional area, weighted by the total cross-sectional area of each size class [Eq. (1) in text]. We obtain $\bar{A}_{c p}$, the VMR inversion estimate of $\bar{A}$ by substituting Eq. (A9) into Eq. (흐) and solving for $\bar{A}$ : 


$$
\bar{A}_{c p}=\frac{\operatorname{Var}\left[c_{p, i}(t)\right]}{E\left[c_{p, i}(t)\right]} \frac{V}{Q_{c}} .
$$

Equation (2) in the text is derived from Eq. (A10), combined with the correction of $V$ for water movement derived in Appendix C. In the event that $Q_{c, i}$ varies strongly with particle class we may replace Eq. (A10) with

$$
\bar{A}_{c p}^{*}=\frac{\operatorname{Var}\left[c_{p, i}(t)\right]}{E\left[c_{p, i}(t)\right]} \frac{V}{Q_{c}^{*}},
$$

where $\bar{A}_{c p}^{*}$ is the mean $A_{i}$, weighted by $\sigma_{c, i}$ and $Q_{c}^{*}$ is a weighted average of $Q_{c, i}$ that depends on a nonlinear interaction between the PSD and $Q_{c, i}$ but must fall between the minimum and maximum $Q_{c, i}$ in suspension.

\section{Appendix B: Correction of $\boldsymbol{V}$ for Inhomogeneities in Sensing Efficiency}

In practice, the contribution of a particle within $V$ to a measurement of $c_{p}(t)$ may depend not only on $\sigma_{c, i}$ and $V$, but also on the position $(x, y, z)$ within $V$. This may result from inhomogeneous illumination of $V$, e.g., by a noncollimated or Gaussian beam source, or by variation in the efficiency of the detection of light scattered from differing positions, e.g., as a function of distance from the detector. Such deviations, combined with the random distribution of particles, will add random variability to measurements of $c_{p}(t)$, introducing a positive bias in $\bar{A}_{c p}$. This bias can be corrected by modifying $V$ as follows.

Let $V_{G}$ be the full geometric sample volume of a sensor, and let a homogeneous scattering medium fill $V_{G}$ (see Table 4 for a list of additional terms introduced in the Appendices). Let $\varepsilon(x, y, z)$ be the signal per unit volume coming from point $x, y, z$, normalized to the total signal. It follows that the spatial integral of $\varepsilon(x, y, z)$ within the limits of $V_{G}$ is equal to one:

$$
\iiint_{V_{G}} \varepsilon(x, y, z) \mathrm{d} x \mathrm{~d} y \mathrm{~d} z=1 .
$$

As a particle moves around the sample volume of a nonhomogeneous sensor, its $\sigma_{c, i}$ will appear to change. If we ignore any variations in $\varepsilon(x, y, z)$ over the spatial extent of a single particle, then we can define $\sigma_{c, i}^{\prime}(x, y, z)$, the "apparent" $\sigma_{c, i}$ at point $x, y, z$, as follows:

$$
\sigma_{c, i}^{\prime}(x, y, z)=\sigma_{c, i} \varepsilon(x, y, z) V_{G} .
$$

We can now treat each particle position as a separate particle class and replace $\overline{\sigma_{c}}$ in Eq. ( $\left.\underline{\mathrm{A} 6}\right)$ with $\overline{\sigma_{c}^{\prime}(x, y, z)}$, yielding

$$
\frac{\operatorname{Var}\left[c_{p}(t)\right]}{E\left[c_{p}(t)\right]}=\frac{\overline{\sigma_{c}^{\prime}(x, y, z)}}{V_{G}},
$$

where $\overline{\sigma_{c}^{\prime}(x, y, z)}$ is defined as

$$
\overline{\sigma_{c}^{\prime}(x, y, z)}=\frac{\sum_{i} E\left[N_{i}(t)\right] \iiint_{V_{G}} \sigma_{c, i}^{\prime 2}(x, y, z) \mathrm{d} x \mathrm{~d} y \mathrm{~d} z}{\sum_{i} E\left[N_{i}(t)\right] \iiint_{V_{G}} \sigma_{c, i}^{\prime}(x, y, z) \mathrm{d} x \mathrm{~d} y \mathrm{~d} z} .
$$

\begin{tabular}{|c|c|c|}
\hline Term & Units & Description \\
\hline $\bar{A}_{c p}^{*}$ & $\mathrm{~m}^{2}$ & Mean $A_{i}$, weighted by $\sigma_{c, i}$ \\
\hline$L_{\mathrm{samp}}$ & $\mathrm{m}$ & Distance sensor moves relative to water during $t_{\mathrm{samp}}$ \\
\hline$L_{t}$ & $\mathrm{~m}$ & Length of $V_{G t}$ in direction of movement \\
\hline$L_{V}$ & $\mathrm{~m}$ & Length of $V_{G}$ in direction of movement \\
\hline$Q_{c}^{*}$ & $\mathrm{~m}^{2}$ & Cross-sectional area of $V_{G}$ in plane perpendicular to direction of motion \\
\hline$t(x)$ & $\mathrm{s}$ & Time that point $x$ in the direction of motion spends in $V_{G}$ \\
\hline$t(x)$ & $\mathbf{S}$ & Time that point $x$ in the direction of motion spends in $V_{G}$ \\
\hline$V_{G}$ & $\mathrm{~m}^{3}$ & Geometric sample volume of a sensor \\
\hline$V_{G t}$ & $\mathrm{~m}^{3}$ & Total geometric volume covered during one sample $\left(t_{\text {samp }}\right)$ of a sensor moving relative to water \\
\hline$V_{t}$ & $\mathrm{~m}^{3}$ & Sample volume weighted over $t_{\mathrm{samp}}$ \\
\hline$x$ & $\mathrm{~m}$ & Position in the direction of movement of sensor relative to water \\
\hline$\varepsilon(x, y, z)$ & $\mathrm{m}^{-3}$ & For a homogeneous scattering medium, signal per unit volume at point $x, y, z$, normalized to total signal \\
\hline$\sigma ; \sigma_{c} ; \sigma_{b b}$ & $\mathrm{~m}^{2}$ & $\begin{array}{l}\text { Optical cross section: Equivalent cross-sectional area of a particle that attenuates }\left(\sigma_{c}\right) \\
\text { or backscatters }\left(\sigma_{b b}\right) 100 \% \text { of incident light }\end{array}$ \\
\hline$\sigma_{c}^{\prime}(x, y, z)$ & $\mathrm{m}^{2}$ & Apparent $\sigma_{c}$ at point $x, y, z$ for sensor with spatially nonhomogeneous sensing efficiency \\
\hline
\end{tabular}

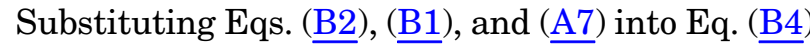
we obtain

$$
\begin{aligned}
\overline{\sigma_{c}^{\prime}(x, y, z)} & =\frac{\sum_{i} E\left[N_{i}(t)\right] \iiint_{V_{G}} \sigma_{c, i}^{2} \varepsilon^{2}(x, y, z) V_{G}^{2} \mathrm{~d} x \mathrm{~d} y \mathrm{~d} z}{\sum_{i} E\left[N_{i}(t)\right] \iiint_{V_{G}} \sigma_{c, i} \varepsilon(x, y, z) V_{G} \mathrm{~d} x \mathrm{~d} y \mathrm{~d} z} \\
& =V_{G} \frac{\sum_{i} E\left[N_{i}(t)\right] \sigma_{c, i}^{2} \iiint_{V_{G}} \varepsilon^{2}(x, y, z) \mathrm{d} x \mathrm{~d} y \mathrm{~d} z}{\sum_{i} E\left[N_{i}(t)\right] \sigma_{c, i} \iiint_{V_{G}} \varepsilon(x, y, z) \mathrm{d} x \mathrm{~d} y \mathrm{~d} z} \\
& =V_{G} \frac{\sum_{i} E\left[N_{i}(t)\right] \sigma_{c, i}^{2} \iiint_{V_{G}} \varepsilon^{2}(x, y, z) \mathrm{d} x \mathrm{~d} y \mathrm{~d} z}{\sum_{i} E\left[N_{i}(t)\right] \sigma_{c, i}} \\
& =\bar{\sigma} V_{G} \iiint_{V_{G}} \varepsilon^{2}(x, y, z) \mathrm{d} x \mathrm{~d} y \mathrm{~d} z .
\end{aligned}
$$

Table 4. Table of Additional Terms in Appendix 
Substituting Eq. (ㅌ5) into Eq. (포), we obtain

$$
\frac{\operatorname{Var}\left[c_{p}(t)\right]}{E\left[c_{p}(t)\right]}=\bar{\sigma}_{c} \iiint_{V_{G}} \varepsilon^{2}(x, y, z) \mathrm{d} x \mathrm{~d} y \mathrm{~d} z
$$

Therefore, in the case of a nonhomogeneous sample volume we can simply define $V$ as a "weighted sample volume", given by

$$
V=\left(\iiint_{V_{G}} \varepsilon^{2}(x, y, z) \mathrm{d} x \mathrm{~d} y \mathrm{~d} z\right)^{-1}
$$

and continue to use Eq. (A6) and all of its derivatives, including the VMR inversion [Eq. (A10)].

For the ac-9 we assume homogeneous sensing efficiency and use $V=V_{G}=5 \mathrm{ml}$ (beam diameter: $0.8 \mathrm{~cm}$; path length: $10 \mathrm{~cm}$ ). We calculate LISST $V$ of $1.9 \mathrm{ml}$ via Eq. (B7) using manufacturer specifications: a Gaussian beam with a waist radius $w$ of $3.5 \mathrm{~mm}$, cut off at a radius of $5 \mathrm{~mm}$, and a path length $L$ of $5 \mathrm{~cm}$. Assuming minimal change in beam width along the path length, we calculate $\varepsilon_{\text {LISST }}$ as a function of radius $r$ from the beam center:

$$
\varepsilon_{\text {LISST }}(r)=\frac{\exp \left(-\frac{2 r^{2}}{w^{2}}\right)}{\iiint_{V_{G}} \exp \left(-\frac{2 r^{2}}{w^{2}}\right) \mathrm{d} x \mathrm{~d} y \mathrm{~d} z}
$$

and substitute into Eq. (B7).

For the ECO BB sensors, we obtained an estimate of $V(0.62 \mathrm{ml})$ using Eq. ( $\underline{\mathrm{B} 7})$, with $\varepsilon_{\mathrm{ECOBB}}$ defined as

$$
\varepsilon_{\mathrm{ECOBB}}(x, y, z)=\frac{P_{d}(x, y, z)}{\Delta V \sum_{x} \sum_{y} \sum_{z} P_{d}(x, y, z)},
$$

where $P_{d}(x, y, z)$ is the power received by the detector of a scattering meter from elemental volume $\Delta V$, centered at point $(x, y, z)$.

We calculated $P_{d}(x, y, z)$ following Sullivan et al. [15], using the Sullivan and Twardowski [34] phase function and the following sensor geometry: angles between center of source or detector cone and sensor face: $49^{\circ}$ and $73^{\circ}$; source and detector cone half angles: $17.5^{\circ}$ and $42.5^{\circ}$; distance between source and detector: $1.7 \mathrm{~cm}$ (Mike Twardowski, pers. comm.). The $P_{d}(x, y, z)$ calculation was modified from Sullivan et al. [15] to correct an error in the original equation, replacing the solid angle of $\Delta V$ viewed from the detector with the solid angle of the detector viewed from $\Delta V$. We also replaced the beam attenuation term with the absorption coefficient of water $\left(0.41 \mathrm{~m}^{-1}\right.$ at $\left.660 \mathrm{~nm}\right)$, because sensitivity analysis showed that attenuation by particles along the path of light in the ECO BB sensors will simultaneously decrease both $V$ and apparent $\sigma_{b b}$, with minimal net effect on the VMR inversion.

\section{Appendix C: Correction for Water Movement}

Each measurement taken by an optical sensor must be integrated over a finite duration $t_{\text {samp }}$. Movement of the water relative to the sensor causes $V_{G t}$, the total geometric volume of water sampled during $t_{\mathrm{samp}}$, to increase beyond $V_{G}$. We may derive $V_{t}$, the weighted volume sampled over $t_{\text {samp }}$, from $V_{G t}$ using Eq. (B7). In this section we find the ratio of $V_{G}$ to $V_{t}$ in terms of $t_{\mathrm{samp}}$ and the residence time $t_{\text {res }}$ of a particle in $V_{G}$ for the simplified case in which the sample moves at a constant velocity in direction $x$ relative to $V_{G}$ and the cross section $A_{y z}$ of $V_{G}$ in the $y-z$ plane is constant along the $x$-dimension. Let us also assume that the sensor response within $V_{G}$ is homogeneous $\left(V_{G}=V\right)$ so that the relative contribution of a point along $x$ to the total signal is determined only by the length of time $t(x)$ that point $x$ spends in $V_{G}$ during

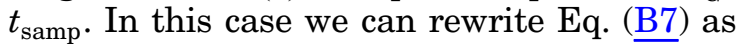

$$
V_{t}=\left(A_{y z} \int_{0}^{L_{t}} \varepsilon^{2}(x) \mathrm{d} x\right)^{-1},
$$

where $L_{t}$ is the length of $V_{G t}$ in the $x$-direction. We can express $\varepsilon(x)$ in terms of $t(x)$ as follows:

$$
\varepsilon(x)=\frac{1}{A_{y z}} \frac{t(x)}{\int_{0}^{L_{t}} t(x) \mathrm{d} x},
$$

and we can divide $L_{t}$ into the length $L_{V}$ of $V_{G}$ in the $x$-direction and the length $L_{\text {samp }}$ that the sensor moves relative to the sample during $t_{\text {samp. }}$. Let us now define a correction factor $\alpha$ in terms of $L_{V}$, $L_{\text {samp }}$, and $t(x)$ as follows:

$$
\alpha=\frac{V}{V_{t}}=\frac{A_{y z} L_{V}}{\left(A_{y z} \int_{0}^{L_{t}} \varepsilon^{2}(x) \mathrm{d} x\right)^{-1}}=\frac{L_{V} \int_{0}^{L_{V}+L_{\mathrm{samp}}} t^{2}(x) \mathrm{d} x}{\left(\int_{0}^{L_{V}+L_{\mathrm{samp}}} t(x) \mathrm{d} x\right)^{2}} .
$$

There are two solutions for Eq. (C3), corresponding to $t_{\text {samp }} \geq t_{\text {res }}$ and $t_{\text {samp }} \leq t_{\text {res }}$. At $\overline{t_{\text {samp }}}=t_{\text {res }}$ the two solutions converge.

For $t_{\mathrm{samp}} \geq t_{\mathrm{res}}$, we can divide $t(x)$ into three sections, defined in relation to $L_{V}$ and $L_{\text {samp: }}$ :

$$
\begin{gathered}
t\left(0 \leq x \leq L_{V}\right)=\frac{t_{\text {res }} x}{L_{V}} \\
t\left(L_{V} \leq x \leq L_{\text {samp }}\right)=t_{\text {res }} \\
t\left(L_{\text {samp }} \leq x \leq L_{\text {samp }}+L_{V}\right)=\frac{t_{\text {res }}\left(L_{\mathrm{samp}}+L_{V}+x\right)}{L_{V}} .
\end{gathered}
$$

The first section represents the water that is within $V_{G}$ at the start of a sample. The second section represents water that is at or beyond the leading edge of $V_{G}$ at the start of the sample and passes through the entire length $L_{V}$ of $V_{G}$ during $t_{\text {samp. }}$. The third section represents water that is within $V_{G}$ at the end of a sample. We can obtain the 
following solutions for the two integrals in Eq. (C3) for $t_{\mathrm{samp}} \geq t_{\mathrm{res}}$ after substituting Eq. (C4):

$$
\begin{aligned}
\int_{0}^{L_{V}+L_{\mathrm{samp}}} t(x) \mathrm{d} x & =\frac{t_{\mathrm{res}} L_{V}}{2}+t_{\mathrm{res}}\left(L_{\mathrm{samp}}-L_{V}\right)+\frac{t_{\mathrm{res}} L_{V}}{2} \\
& =t_{\mathrm{res}}\left(L_{V}+L_{\mathrm{samp}}-L_{V}\right) \\
& =t_{\mathrm{res}} L_{\mathrm{samp}}
\end{aligned}
$$

and

$$
\begin{aligned}
\int_{0}^{L_{V}+L_{\mathrm{samp}}} t^{2}(x) \mathrm{d} x & =\frac{t_{\mathrm{res}}^{2} L_{V}}{3}+t_{\mathrm{res}}^{2}\left(L_{\mathrm{samp}}-L_{V}\right)+\frac{t_{\mathrm{res}}^{2} L_{V}}{3} \\
& =t_{\mathrm{res}}^{2}\left(\frac{2 L_{V}}{3}+L_{\mathrm{samp}}-L_{V}\right) \\
& =t_{\mathrm{res}}^{2}\left(L_{\mathrm{samp}}-\frac{L_{V}}{3}\right) .
\end{aligned}
$$

Substituting Eq. (C5) and Eq. ( $\underline{\text { C6 }) ~ i n t o ~ E q . ~(C 3) ~ w e ~}$ obtain the solution

$$
\begin{aligned}
\alpha & =\frac{L_{V} t_{\mathrm{res}}^{2}\left(L_{\mathrm{samp}}-\frac{L_{V}}{3}\right)}{t_{\mathrm{res}}^{2} L_{\mathrm{samp}}^{2}}=\frac{L_{V}\left(L_{\mathrm{samp}}-\frac{L_{V}}{3}\right)}{L_{\mathrm{samp}}^{2}} \\
& =\frac{L_{V}}{L_{\mathrm{samp}}}-\frac{1}{3}\left(\frac{L_{V}}{L_{\mathrm{samp}}}\right)^{2} .
\end{aligned}
$$

We can find $\alpha$ in a similar fashion for $t_{\text {samp }} \leq t_{\text {res }}$ as well. In this case, $t(x)$ becomes

$$
\begin{aligned}
& t\left(0 \leq x \leq L_{\mathrm{samp}}\right)=\frac{t_{\mathrm{samp}} x}{L_{S}}, \\
& t\left(L_{\mathrm{samp}} \leq x \leq L_{V}\right)=t_{\mathrm{samp}}, \\
& t\left(L_{V} \leq x \leq L_{\mathrm{samp}}+L_{V}\right)=\frac{t_{\mathrm{samp}}\left(L_{\mathrm{samp}}+L_{V}+x\right)}{L_{\mathrm{samp}}} .
\end{aligned}
$$

Equation ( $\underline{\mathrm{C} 8})$ is identical to Eq. (3), except that all instances of $L_{\text {samp }}$ and $L_{V}$ have been reversed, and

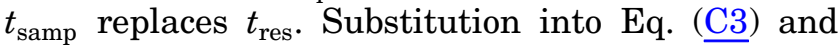
integration in this case yields

$$
\alpha=1-\frac{1}{3} \frac{L_{\mathrm{samp}}}{L_{V}} .
$$

Let us now define $\tau$ as the ratio of $L_{V}$ to $L_{\mathrm{samp}}$ and note that $\tau$ must also equal the ratio of $t_{\text {res }}$ to $t_{\mathrm{samp}}$ [Eq. () in text]:

$$
\tau=\frac{L_{V}}{L_{\mathrm{samp}}}=\frac{t_{\mathrm{res}}}{t_{\mathrm{samp}}}
$$

We can now combine Eqs. (C7) and (C9) and express them in terms of $\tau$ :

$$
\alpha(\tau)= \begin{cases}1-(3 \tau)^{-1}, & \text { if } \tau \geq 1 \\ \tau-\frac{\tau^{2}}{3}, & \text { if } \tau \leq 1\end{cases}
$$

Note that $\alpha(\tau)$ is continuous for $\tau=1$.

In order to obtain the full VMR inversion equation, corrected for water movement, we must replace $V$ with $V_{t}$ in Eq. (A10). The expression $V / \alpha(\tau)$ may then be substituted for $V_{t}$ [Eq. (C3)] to obtain Eq. (2) from the text.

\section{Appendix D: Effect of Particle-Particle Shading}

Implicit in Eq. (A1) and all derivations that follow is the assumption that the contribution of each particle to $c_{p}$ is not changed by the presence of other particles. This is a fundamental assumption of any measurement of an inherent optical property such as $c_{p}$ or $b_{b p}$. However, violation of this assumption will affect the VMR inversion differently than it affects $E\left[c_{p}\right]$ or $E\left[b_{b p}\right]$, so a brief discussion is warranted.

In a beam transmissometer, the light attenuated by a particle is detected by the reduction in source light that reaches the sensor. If this particle is in the shadow of another particle, this reduction in detected light is decreased. If particles are randomly distributed in space, the calculation of $c_{p}$ takes into account the mean effect of shading on the detected signal. However, random variations around this meannot accounted for by the VMR inversion-will cause a positive bias when present by adding to the variance. The magnitude of variations in $c_{p}$ caused by random particle-particle shading will depend on the concentration, size, shape, and transparency of particles in suspension. The time scale of these variations will depend on the particles' size and relative speed. Shifrin's (1988) particle size inversion relies on the same fundamental assumptions as the VMR inversion, but additionally incorporates the effect of variation in particle-particle shading for completely opaque spherical particles (i.e., the maximum possible effect). Therefore, if all other assumptions of both size inversions are met, but if particles are not completely opaque and spherical or if some of the fluctuations caused by particle-particle shading occur too rapidly to be detected by the transmissometer, then the true mean particle size will fall somewhere in between the estimates of the Shifrin inversion and the VMR inversion. The effect of particle-particle shading on the validation dataset presented in this paper is discussed in Subsection 4.C.

A scattering meter detects light that travels from the light source to a particle, where it is scattered and then to the sensor. Absorption by other particles along this path will decrease the scattered light that is detected. At very high particle concentrations, or for scattering meters with long path lengths, an absorption correction factor may be necessary to correct the mean scattering measurement. If uncorrected scattering measurements are used, absorption will induce a low bias in the VMR inversion. If an absorption correction is applied to scattering measurements before the VMR inversion, accounting for 
mean shading, there may be a small high bias in the VMR inversion due to variability in this shading. While absorption was not measured during this study, any particle-particle shading effect was likely minimal due to the short path length of the backscattering instruments.

\section{References}

1. L. Dickie, S. Kerr, and P. Boudreau, "Size-dependent processes underlying regularities in ecosystem structure," Ecol. Monogr. 57, 233-250 (1987)

2. S. Jennings and K. J. Warr, "Smaller predator-prey body size ratios in longer food chains," Proc. R. Soc. B 270, 1413-1417 (2003).

3. V. S. Smetacek, "Role of sinking in diatom life-history cycles: ecological, evolutionary and geological significance," Mar. Biol. 84, 239-251 (1985)

4. A. L. Alldredge and M. W. Silver, "Characteristics, dynamics and significance of marine snow," Prog. Oceanogr. 20, 41-82 (1988).

5. V. L. Asper and W. O. Smith, "Abundance, distribution and sinking rates of aggregates in the Ross Sea, Antarctica," Deep Sea Res., Part I 50, 131-150 (2003).

6. G. A. Jackson, "Effect of mixed layer depth on phytoplankton removal by coagulation and on the critical depth concept," Deep Sea Res., Part I 55, 766-776 (2008).

7. G. Fischer and G. Karakas, "Sinking rates and ballast composition of particles in the Atlantic Ocean: implications for the organic carbon fluxes to the deep ocean," Biogeosciences 6, 85-102 (2009).

8. J. C. Winterwerp and W. G. M. van Kesteren, Introduction to the Physics of Cohesive Sediment Dynamics in the Marine Environment (Elsevier, 2004).

9. G. C. Chang, T. D. Dickey, and A. J. Williams, "Sediment resuspension over a continental shelf during Hurricanes Edouard and Hortense," J. Geophys. Res. 106, 9517-9531 (2001).

10. J. R. Gray and J. W. Gartner, "Technological advances in suspended-sediment surrogate monitoring," Water Resour. Res. 45 (2009).

11. K. J. Curran, P. S. Hill, and T. G. Milligan, "Fine-grained suspended sediment dynamics in the Eel River flood plume," Cont. Shelf Res. 22, 2537-2550 (2002).

12. K. S. Shifrin, B. Z. Moroz, and A. N. Sakharov, "Determination of characteristics of a dispersion medium based on its transparency data," Dokl. Akad. Nauk SSSR 199, 589-591 (1971).

13. K. S. Shifrin, "The method of fluctuations," in Physical Optics of Ocean Water (American Institute of Physics, 1988), pp. 224-232.

14. W. Slade, E. Boss, and C. Russo, "Effects of particle aggregation and disaggregation on their inherent optical properties," Opt. Express 19, 7945-7959 (2011).

15. J. M. Sullivan, M. Twardowski, J. R. Zaneveld, and C. Moore, "Measuring optical backscattering in water" in Light Scattering Reviews 7, A. A. Kokhanovsky, ed. (Springer, 2013), pp. 189-224.

16. J. E. Freund, Mathematical Statistics, 2nd ed. (Prentice-Hall, 1971), pp. 212-216.

17. W. D. Gardner, M. J. Richardson, and W. O. Smith, "Seasonal patterns of water column particulate organic carbon and fluxes in the Ross Sea, Antarctica," Deep Sea Res., Part II 47, 3423-3449 (2000).

18. J. K. B. Bishop and T. J. Wood, "Particulate matter chemistry and dynamics in the twilight zone at VERTIGO ALOHA and K2 sites," Deep Sea Res., Part I 55, 1684-1706 (2008).

19. N. Briggs, M. J. Perry, I. Cetinić, C. Lee, E. D’Asaro, A. M. Gray, and E. Rehm, "High-resolution observations of aggregate flux during a sub-polar North Atlantic spring bloom," Deep Sea Res., Part I 58, 1031-1039 (2011).

20. G. Dall'Olmo, E. Boss, M. J. Behrenfeld, and T. K. Westberry, "Particulate optical scattering coefficients along an Atlantic Meridional Transect," Opt. Express 20, 21532-21551 (2012).

21. E. Boss, W. H. Slade, M. Behrenfeld, and G. Dall'Olmo, "Acceptance angle effects on the beam attenuation in the ocean," Opt. Express 17, 1535-1550 (2009).

22. C. F. Bohren and D. R. Huffman, Absorption and Scattering of Light by Small Particles (Wiley, 1983).

23. E. Boss, W. S. Pegau, M. Lee, M. Twardowski, E. Shybanov, G. Korotaev, and F. Baratange, "Particulate backscattering ratio at LEO 15 and its use to study particle composition and distribution,” J. Geophys. Res. 109, C01014 (2004).

24. M. S. Twardowski, E. Boss, J. B. Macdonald, W. S. Pegau, A. H. Barnard, and J. R. V. Zaneveld, "A model for estimating bulk refractive index from the optical backscattering ratio and the implications for understanding particle composition in case I and case II waters," J. Geophys. Res. 106, 14129-14142 (2001).

25. M. Babin, A. Morel, V. Fournier-Sicre, F. Fell, and D. Stramski, "Light scattering properties of marine particles in coastal and open ocean waters as related to the particle mass concentration," Limnol. Oceanogr. 48, 843-859 (2003).

26. W. Dietrich, "Settling velocity of natural particles," Water Resour. Res. 18, 1615-1626 (1982).

27. E. Boss, M. S. Twardowski, and S. Herring, "Shape of the particulate beam attenuation spectrum and its inversion to obtain the shape of the particulate size distribution," Appl. Opt. 40, 4885-4893 (2001).

28. T. S. Kostadinov, D. A. Siegel, and S. Maritorena, "Retrieval of the particle size distribution from satellite ocean color observations," J. Geophys. Res. 114, C09015 (2009).

29. R. A. Reynolds, D. Stramski, V. M. Wright, and S. B. Woźniak, "Measurements and characterization of particle size distributions in coastal waters," J. Geophys. Res. 115, 1-19 (2010).

30. O. Mikkelsen, T. Milligan, and P. Hill, "The influence of schlieren on in situ optical measurements used for particle characterization," Limnol. Oceanogr. 6, 133-143 (2008).

31. H. Loisel, J. M. Nicolas, A. Sciandra, D. Stramski, and A. Poteau, "Spectral dependency of optical backscattering by marine particles from satellite remote sensing of the global ocean," J. Geophys. Res. 111, C09024 (2006).

32. M. J. Behrenfeld and E. Boss, "Beam attenuation and chlorophyll concentration as alternative optical indices of phytoplankton biomass," J. Mar. Res. 64, 431-451 (2006).

33. W. Clavano, E. Boss, and L. Karp-Boss, "Inherent optical properties of non-spherical marine-like particles-from theory to observation," Oceanogr. Mar. Biol. 45, 1-38 (2007).

34. J. M. Sullivan and M. S. Twardowski, "Angular shape of the oceanic particulate volume scattering function in the backward direction," Appl. Opt. 48, 6811-6819 (2009). 